\title{
Composite Dark Matter and a horizontal symmetry
}

\author{
Alexandre Carvunis, Diego Guadagnoli, Méril Reboud and Peter Stangl \\ Laboratoire d'Annecy-le-Vieux de Physique Théorique UMR5108, \\ Université de Savoie Mont-Blanc et CNRS, \\ B.P. 110, F-74941, Annecy Cedex, France \\ E-mail: carvunis@lapth.cnrs.fr, diego.guadagnoli@lapth.cnrs.fr, \\ meril.reboud@tum.de, stangl@itp.unibe.ch
}

ABStRaCT: We present a model of composite Dark Matter (DM), in which a new QCD-like confining "hypercolor" sector generates naturally stable hyperbaryons as DM candidates and at the same time provides mass to new weakly coupled gauge bosons $H$ that serve as DM mediators, coupling the hyperbaryons to the Standard Model (SM) fermions. By an appropriate choice of the $H$ gauge symmetry as a horizontal SU $(2)_{h}$ SM flavor symmetry, we show how the $H$ gauge bosons can be identified with the horizontal gauge bosons recently put forward as an explanation for discrepancies in rare $B$-meson decays. We find that the mass scale of the $H$ gauge bosons suggested by the DM phenomenology intriguingly agrees with the one needed to explain the rare $B$-decay discrepancies.

Keywords: Beyond Standard Model, Chiral Lagrangians, Cosmology of Theories beyond the SM, Technicolor and Composite Models

ARXIV EPrint: 2007.11931 


\section{Contents}

1 Introduction 1

2 Model 3

2.1 NGBs and baryon Lagrangian: the DM sector 6

3 Dark-Matter direct detection $\quad 10$

4 Dark Matter relic density 12

4.1 Calculation of $\left\langle\sigma_{\text {eff }} v\right\rangle$ and $J \quad 13$

$\begin{array}{lll}4.2 \Omega_{0} h^{2} \text { prediction } & 16\end{array}$

$\begin{array}{lll}4.3 & \text { Comments on Dark-Matter indirect detection } & 17\end{array}$

$\begin{array}{lll}5 & \text { Conclusions } & 17\end{array}$

$\begin{array}{ll}\text { A Mixing between SM and VL fermions } & 19\end{array}$

B Possible UV completions $\quad 20$

B.1 Scalar extension 20

$\begin{array}{lll}\text { B.2 Gauge extension } & 21\end{array}$

\section{Introduction}

New massive particles that only weakly interact with the Standard Model (SM) particles, the so-called weakly interacting massive particles (WIMPs), are among the most promising explanations of Dark Matter (DM) $[1,2]$. One main challenge in constructing viable WIMP models is the requirement that the DM candidate has to be stable on time scales of the order of the age of the Universe. Quite generically, such stability requires the presence of an approximately unbroken global symmetry, one famous example being $R$-parity in supersymmetric DM models. In the SM, the only global symmetry that provides stability to a heavy particle is the $\mathrm{U}(1)_{B}$ baryon symmetry. It protects the lightest baryonic bound state from decaying to leptons, thus providing the proton with a mean lifetime of more than $10^{29}$ years [3]. An immediate question is then whether in an extension of the SM, a symmetry similar to the $\mathrm{U}(1)_{B}$ baryon symmetry could provide stability to a DM candidate. Such an extension can be given by a new QCD-like confining "hypercolor" (HC) sector, in which hyperquarks are charged under an accidental $\mathrm{U}(1)_{\mathrm{HB}}$ hyperbaryon symmetry. These hyperquarks form baryonic bound states, the hyperbaryons, which are rendered stable by carrying non-zero $\mathrm{U}(1)_{\mathrm{HB}}$ charges and are thus potential DM candidates. ${ }^{1}$

\footnotetext{
${ }^{1}$ Several models with a composite DM made stable by a similar symmetry mechanism have been proposed. Those we are aware of include [4-26]. For a review, see [27].
} 
In addition to hyperbaryons, such a construction also leads to hypermeson bound states formed by a hyperquark and an antihyperquark. Carrying zero U(1) HB charge, the hypermesons are in general unstable. Furthermore, without an explicit breaking of the hyperquarks' chiral symmetry, the lightest hypermesons are massless Nambu-Goldstone bosons (NGBs). The appearance of phenomenologically unacceptable massless scalars can be avoided in two ways. Lagrangian terms that explicitly break the chiral symmetry can generate a potential for the NGBs, turning them into massive pseudo NGBs (pNGBs) [28]. An example are the pions in QCD, which are massive because the quark mass terms explicitly break the quarks' chiral symmetry. The second possibility to avoid massless NGBs is the gauging of an appropriate subgroup of the chiral symmetry. In this case, the corresponding gauge bosons become massive due to the Higgs mechanism and the wouldbe NGBs become the gauge bosons' longitudinal degrees of freedom. Among these two possibilities, the latter one is particularly interesting in the context of a DM model since the gauging of a subgroup of the chiral symmetry implies that the corresponding gauge bosons, which we denote by $H$, couple to the hyperbaryon DM candidates. If these $H$ gauge bosons also couple to some of the SM particles, they then not only solve the problem of massless NGBs, but they could also serve as mediators to a dark sector naturally endowed with a stability mechanism.

Interestingly, in this setup, the masses of the hyperbaryons $\mathcal{X}$ are related to those of the gauge bosons $H$ by

$$
m_{\chi} \approx 10 v_{\mathrm{HC}} \quad \text { where } \quad v_{\mathrm{HC}}=2 m_{H} / g_{H}
$$

with $g_{H}$ the $H$ gauge coupling and $v_{\mathrm{HC}}$ the NGB decay constant associated to the dynamical breaking of the chiral symmetry by a hyperquark condensate. Unitarity arguments suggest that the mass of a thermal relic DM candidate cannot exceed $340 \mathrm{TeV}$ [29]. Taking the hyperbaryons $\mathcal{X}$ as DM candidates, eq. (1.1) then implies

$$
v_{\mathrm{HC}} \lesssim 34 \mathrm{TeV}
$$

This puts the $H$ gauge bosons in the mass range probed by flavor physics experiments. Intriguingly, current data on rare $B$-meson decays shows deviations from the SM predictions in several measurements, most notably in the theoretically clean lepton flavor universality ratios $R_{K^{(*)}}$ [30-33], which hint at new physics (NP) at a scale well compatible with eq. (1.2) [34]. The discrepancies in rare $B$ decays can be explained by NP contributions to the $b \rightarrow$ sll transition [35-40], which can be generated at tree level either by leptoquarks or SM-neutral bosons. An immediate question is whether the $H$ gauge bosons can play the role of such tree-level mediators contributing to the $b \rightarrow$ sll transition. They could be leptoquarks only if the chiral symmetries of the hyperquarks contained at least the SM $\mathrm{SU}(3)_{C}$ and $\mathrm{U}(1)_{Y}$ groups as subgroups. However, in this case the hyperbaryons would in general not be SM-neutral DM candidates. ${ }^{2}$ On the other hand, in the case of SM-neutral $H$

\footnotetext{
${ }^{2}$ In a model featuring a leptoquark that explains the $B$-discrepancies and gets its mass from a QCD-like hypercolor sector, SM-neutral hyperbaryons could be formed e.g. from two types of hyperquarks transforming in different representations of the HC gauge group [41].
} 
gauge bosons, the hyperbaryons are naturally SM-neutral, thus allowing them to be viable DM candidates. A main challenge for models explaining the $b \rightarrow$ sll discrepancies in terms of SM-neutral tree-level mediators are too large NP contributions to $B_{s}-\bar{B}_{s}$ mixing [44]. However, such large contributions can be avoided if the mediators are the gauge bosons of a gauged horizontal $\mathrm{SU}(2)_{h}$ flavor symmetry [45]. We are thus led to a well-motivated model with the following features:

- A new QCD-like confining hypercolor sector generates baryonic bound states of hyperquarks, the hyperbaryons, which are SM-neutral and stable and are thus natural DM candidates.

- Massless NGBs are avoided by gauging an appropriate subgroup of the hyperquarks' chiral symmetry, resulting in massive gauge bosons $H$.

- The $H$ gauge bosons are coupled to SM fields by identifying the $H$ gauge group with the diagonal subgroup of part of the hyperquarks' chiral symmetry and a horizontal $\mathrm{SU}(2)_{h}$ flavor symmetry of SM fermions. This allows the $H$ gauge bosons to address the discrepancies in rare $B$-decays as proposed in [45].

- The $H$ gauge bosons couplings to hyperquarks and SM fermions as described above allows them to play the role of the DM mediators, opening the possibility of a fully calculable DM freeze-out scenario with the hyperbaryons as DM candidates.

The remaining parts of this paper analyzes the DM phenomenology of this construction. The details of the model are discussed in section 2. In section 3 we investigate possible bounds from DM direct detection. Our prediction for the DM relic density and a discussion of the viable parameter space allowing for an explanation of the observed relic density are presented in section 4 . In section 5 we conclude.

\section{Model}

In order to illustrate the idea in our paper, let us start from a known example: twoflavor QCD. In the absence of explicit mass terms, the left- and right-handed $u$ and $d$ quarks transform as doublets, $\left(u_{L}, d_{L}\right)^{T}$ and $\left(u_{R}, d_{R}\right)^{T}$, under the global symmetry groups $\mathrm{SU}(2)_{L}$ and $\mathrm{SU}(2)_{R}$, respectively. The global chiral symmetry group is therefore $\mathcal{G}_{\chi}=$ $\mathrm{SU}(2)_{L} \times \mathrm{SU}(2)_{R}$. This symmetry is spontaneously broken to $\mathcal{H}_{V}=\mathrm{SU}(2)_{V}$ by the quark condensate $\left\langle\bar{u}_{L} u_{R}+\bar{d}_{L} d_{R}+\right.$ H.c. $\rangle \neq 0$ at the scale $f_{\pi} \simeq 93 \mathrm{MeV}$. The consequence are Goldstone bosons spanning the coset space $\mathcal{G}_{\chi} / \mathcal{H}_{V}$, the known pions. As well known, the $\mathrm{SU}(2)_{L}$ group is actually gauged, and this gauge symmetry is spontaneously broken also by the Higgs vev $v=246 \mathrm{GeV}$. Because of the hierarchy $f_{\pi} \ll v$, the pions contribute negligibly to the longitudinal degrees of freedom of the $\mathrm{SU}(2)_{L}$ gauge bosons.

Pursuing the analogy, one may introduce two flavors of new massless vector-like fermions, $\mathcal{F}_{2}$ and $\mathcal{F}_{3}$, charged under a new strong force, corresponding to the gauge group $\mathcal{G}_{\mathrm{HC}} \equiv \mathrm{SU}(N)_{\mathrm{HC}}$ that will be denoted by hypercolor. ${ }^{3}$ In order to be able to use known

\footnotetext{
${ }^{3}$ Following existing literature, this naming denotes vector-like confinement, or a new chiral-symmetric QCD-like force, see in particular [46-50].
} 
results from QCD for otherwise incalculable quantities (cf. section 4.1), we fix $N=3$. Just like in two-flavor QCD, these new fermions transform as doublets $\mathcal{F}_{L}=\left(\mathcal{F}_{2 L}, \mathcal{F}_{3 L}\right)^{T}$ and $\mathcal{F}_{R}=\left(\mathcal{F}_{2 R}, \mathcal{F}_{3 R}\right)^{T}$ under the global symmetry groups $\mathrm{SU}(2)_{L}^{\mathcal{F}}$ and $\mathrm{SU}(2)_{R}^{\mathcal{F}}$, respectively. The chiral condensate ${ }^{4}\left\langle\overline{\mathcal{F}}_{2 L} \mathcal{F}_{2 R}+\overline{\mathcal{F}}_{3 L} \mathcal{F}_{3 R}+\right.$ H.c. $\rangle \propto v_{\mathrm{HC}}^{3}$ breaks the chiral symmetry group $G^{\mathcal{F}} \equiv \mathrm{SU}(2)_{L}^{\mathcal{F}} \times \mathrm{SU}(2)_{R}^{\mathcal{F}}$ to the diagonal $\mathrm{SU}(2)_{V}^{\mathcal{F}}$. Then, if either $\mathrm{SU}(2)_{L}^{\mathcal{F}}$ or $\mathrm{SU}(2)_{R}^{\mathcal{F}}$ is gauged, this gauge symmetry, to be denoted by $\mathrm{SU}(2)_{h}$, is also spontaneously broken by the chiral condensate. As a consequence, the $\mathrm{SU}(2)_{h}$ gauge bosons $H_{\mu}^{a}$ acquire mass, and the would-be Goldstone bosons of the spontaneously broken group supply the $H_{\mu}^{a}$ longitudinal polarizations. One therefore ends up with a $H_{\mu}^{a}$ boson triplet, whose transverse polarizations are elementary, while their longitudinal ones are composite $\overline{\mathcal{F}} \mathcal{F}$ bound states.

Concretely, it is $\mathrm{SU}(2)_{R}^{\mathcal{F}}$ that we choose to gauge, and identify with $\mathrm{SU}(2)_{h}$, the horizontal gauge symmetry introduced in ref. [45]. There, it is assumed that $2^{\text {nd }}$ and $3^{\text {rd }}-$ generation left-handed SM fermions transform as doublets under $\mathrm{SU}(2)_{h}$

$$
Q^{\prime} \equiv\left(q_{2}^{\prime}, q_{3}^{\prime}\right)^{T}, \quad L^{\prime} \equiv\left(l_{2}^{\prime}, l_{3}^{\prime}\right)^{T}
$$

with $q_{2,3}^{\prime}$ and $l_{2,3}^{\prime}$ denoting the SM quark and lepton doublets of generation index $2,3 .^{5}$ Such construction was advocated in ref. [45] to account for discrepancies in $b \rightarrow s \ell$. More quantitatively, the $\mathrm{SU}(2)_{h} \times \mathrm{SU}(2)_{L}^{\mathcal{F}}$ symmetry-breaking scale $v_{\mathrm{HC}}$ can be related to the effective scale pointed to by the SM $b \rightarrow$ sll Hamiltonian $\mathcal{H}_{\mathrm{SM}}^{b \rightarrow s}$. Such scale bounds the ratio between the mass of the horizontal bosons $m_{H}$, and the coupling $g_{H}$ of the horizontal symmetry [45]. Using $m_{H}=g_{H} v_{\mathrm{HC}} / 2$, this translates into the indicative range

$$
v_{\mathrm{HC}} \in[10,30] \mathrm{TeV},
$$

which is nicely compatible with the bound in eq. (1.2).

Due to the fact that LH SM fermions are charged under SU $(2)_{h}$ while RH SM fermions are not, the usual Higgs Yukawa couplings are forbidden by gauge invariance and can only arise after the spontaneous breaking of $\mathrm{SU}(2)_{h}$. Two options to accomplish this are:

- The SM Higgs is part of a larger scalar multiplet that transforms non-trivially under $\mathrm{SU}(2)_{h}$ in such a way that it can have gauge-invariant Yukawa couplings with LH and RH SM fermions. This option would lead to an enlarged Higgs sector.

- The SM fermions mix with new fermions that are vector-like (VL) under the SM gauge group and transform non-trivially under $\mathrm{SU}(2)_{h}$ in such a way that gaugeinvariant Higgs Yukawa couplings between the new VL fermions and SM fermions are possible. This option allows for a minimal Higgs sector but requires an enlarged fermion sector.

In the following, we focus on the latter option since an enlarged fermion sector is required also for another reason. In a model containing only the SM fermions and the hyperquarks

\footnotetext{
${ }^{4}$ Note that in QCD one has $\langle\bar{u} u+\bar{d} d\rangle=-2 B f^{2} \approx-16 \pi f^{3}$.

${ }^{5}$ As we will discuss below, the prime denotes SM fields before EW-scale flavor mixing and also before hypercolor-scale mixing with vector-like new fermions.
} 
$\mathcal{F}$, there would be an $\mathrm{SU}(2)_{h}$ gauge anomaly in case of an $\mathrm{SU}(N)_{\mathrm{HC}}$ group with odd $N$ [55], in particular for our assumption $N=3$. In this case, the $\mathrm{SU}(2)_{h}$ gauge anomaly has to be canceled by an enlarged fermion sector containing an odd number of $\mathrm{SU}(2)_{h}$ doublets. We therefore introduce new RH fermions $\Psi_{R}$ with the same SM quantum numbers as one generation of RH SM fermions but transforming as doublets under $\mathrm{SU}(2)_{h}$. In addition, we introduce two sets of new LH fermions $\Psi_{L}^{i}$ with the same SM quantum numbers as $\Psi_{R}$ but transforming trivially under $\mathrm{SU}(2)_{h}$. Furthermore, we assume the presence of an 'extended' hypercolor (EHC) sector that generates at least one of the four-fermion operators ${ }^{6}$

$$
\frac{c_{i j}}{\Lambda_{\mathrm{EHC}}^{2}}\left(\bar{\Psi}_{L}^{i} \Psi_{R}\right)\left(\overline{\mathcal{F}}_{R} \mathcal{F}_{L}^{j}\right)+\text { h.c. }, \quad \frac{\tilde{c}_{i j}}{\Lambda_{\mathrm{EHC}}^{2}}\left(\bar{\Psi}_{L}^{i} \Psi_{R}\right) \epsilon_{h}\left(\overline{\mathcal{F}}_{L}^{j} \mathcal{F}_{R}\right)+\text { h.c. },
$$

which both generate a vector-like mass term for $\Psi$ once the hyperquarks condense. Here, $\epsilon_{h}$ is the anti-symmetric tensor of $\mathrm{SU}(2)_{h}$. Some speculations about how the effective interactions in eqs. (2.3), as well as the Yukawa terms in eq. (2.4) below, may arise from a UV-complete model are collected in appendix B.

All fermions in the model and their quantum numbers are collected in table 1. The possible gauge invariant and renormalizable couplings between SM fermions, new fermions $\Psi$, and the Higgs field $\varphi$ are given by

$$
\begin{aligned}
\mathscr{L} \supset & -\Delta_{u}^{i k} \bar{\Psi}_{L}^{\prime u i} u_{R}^{\prime k}-\Delta_{d}^{i k} \bar{\Psi}_{L}^{\prime d i} d_{R}^{\prime k}-\Delta_{e}^{i k} \bar{\Psi}_{L}^{\prime e i} e_{R}^{\prime k} \\
& -y_{u}^{k} \bar{q}_{L}^{\prime 1} \tilde{\varphi} u_{R}^{\prime k}-y_{d}^{k} \bar{q}_{L}^{\prime 1} \varphi d_{R}^{\prime k}-y_{e}^{k} \bar{l}_{L}^{\prime 1} \varphi e_{R}^{\prime k} \\
& -y_{U} \bar{Q}_{L}^{\prime} \tilde{\varphi} \Psi_{R}^{\prime u}-y_{D} \bar{Q}_{L}^{\prime} \varphi \Psi_{R}^{\prime d}-y_{E} \bar{L}_{L}^{\prime} \varphi \Psi_{R}^{\prime e},
\end{aligned}
$$

where the coefficients $\Delta$ denote mass-mixing terms between the $\Psi$ and SM fields, and the indices $i \in\{2,3\}, k \in\{1,2,3\}$.

By way of SM- and $\Psi$-fermion field redefinitions in flavor space, the above mixing Lagrangian leads to the ordinary SM Yukawa terms. The detailed derivation is presented in the appendix. The DM phenomenology to follow depends on the Yukawa sector only through the matrices leading from the primed basis to the mass eigenbasis for quarks. In fact, quarks only communicate with the DM sector through the exchange of horizontal bosons, i.e. through the interaction introduced explicitly in eq. (3.1).

A further comment is in order. Although the horizontal symmetry involves only LH fermions of the $2^{\text {nd }}$ and $3^{\text {rd }}$ generation, the field redefinitions necessary to obtain the fermion mass eigenbasis will induce also contributions involving the first generation, as discussed in ref. [45]. In turn, the latter induce potentially large shifts in meson-mixing observables such as $K^{0}-\bar{K}^{0}$ and $D^{0}-\bar{D}^{0}$.Specifically, in ref. [45] it is argued that flavour mixing can be arranged to have all flavour-changing effects in the up sector, resulting in mixing contributions to $D^{0}-\bar{D}^{0}$ only. In such a scenario, the resulting new-physics contribution to the mixing observable $x_{D}$ is a factor 10 above the measured value $x_{D} \simeq 10^{-2}$ [59]. We remark however that the corresponding SM result for $x_{D} \equiv \Delta m_{D} / \Gamma$ suffers from very large uncertainties, and could be several percent, because on the one hand $y_{D} \equiv \Delta \Gamma /(2 \Gamma)$ is

\footnotetext{
${ }^{6}$ Here we use the denomination 'extended' similarly as in extended technicolor [56, 57], namely to denote dynamics designed to account for fermion masses.
} 


\begin{tabular}{|c|c|c|c|c|c|}
\hline Field & $\mathrm{SU}(3)_{\mathrm{HC}}$ & $\mathrm{SU}(2)_{h}$ & $\mathrm{SU}(3)_{c}$ & $\mathrm{SU}(2)_{L}$ & $\mathrm{U}(1)_{Y}$ \\
\hline \hline$Q_{L}^{\prime}$ & $\mathbf{1}$ & $\mathbf{2}$ & $\mathbf{3}$ & $\mathbf{2}$ & $+1 / 6$ \\
$L_{L}^{\prime}$ & $\mathbf{1}$ & $\mathbf{2}$ & $\mathbf{1}$ & $\mathbf{2}$ & $-1 / 2$ \\
$q_{L}^{\prime 1}$ & $\mathbf{1}$ & $\mathbf{1}$ & $\mathbf{3}$ & $\mathbf{2}$ & $+1 / 6$ \\
$\ell_{L}^{\prime 1}$ & $\mathbf{1}$ & $\mathbf{1}$ & $\mathbf{1}$ & $\mathbf{2}$ & $-1 / 2$ \\
$u_{R}^{\prime 1,2,3}$ & $\mathbf{1}$ & $\mathbf{1}$ & $\mathbf{3}$ & $\mathbf{1}$ & $+2 / 3$ \\
$d_{R}^{\prime 1,2,3}$ & $\mathbf{1}$ & $\mathbf{1}$ & $\mathbf{3}$ & $\mathbf{1}$ & $-1 / 3$ \\
$e_{R}^{\prime 1,2,3}$ & $\mathbf{1}$ & $\mathbf{1}$ & $\mathbf{1}$ & $\mathbf{1}$ & -1 \\
\hline \hline$\Psi_{R}^{\prime u}$ & $\mathbf{1}$ & $\mathbf{2}$ & $\mathbf{3}$ & $\mathbf{1}$ & $+2 / 3$ \\
$\Psi_{R}^{\prime d}$ & $\mathbf{1}$ & $\mathbf{2}$ & $\mathbf{3}$ & $\mathbf{1}$ & $-1 / 3$ \\
$\Psi_{R}^{\prime e}$ & $\mathbf{1}$ & $\mathbf{2}$ & $\mathbf{1}$ & $\mathbf{1}$ & -1 \\
$\Psi_{L}^{\prime u 2,3}$ & $\mathbf{1}$ & $\mathbf{1}$ & $\mathbf{3}$ & $\mathbf{1}$ & $+2 / 3$ \\
$\Psi_{L}^{\prime d 2,3}$ & $\mathbf{1}$ & $\mathbf{1}$ & $\mathbf{3}$ & $\mathbf{1}$ & $-1 / 3$ \\
$\Psi_{L}^{\prime e 2,3}$ & $\mathbf{1}$ & $\mathbf{1}$ & $\mathbf{1}$ & $\mathbf{1}$ & -1 \\
\hline \hline $\mathcal{F}_{R}$ & $\mathbf{3}$ & $\mathbf{2}$ & $\mathbf{1}$ & $\mathbf{1}$ & 0 \\
$\mathcal{F}_{L}^{2,3}$ & $\mathbf{3}$ & $\mathbf{1}$ & $\mathbf{1}$ & $\mathbf{1}$ & 0 \\
\hline
\end{tabular}

Table 1. Quantum numbers of SM-like fermions (first block), new vector-like fermions $\Psi$ (second block), and hyperquarks $\mathcal{F}$ (third block).

naturally of order few $\times 10^{-2}$ (see e.g. [60]), and on the other hand $\Delta m_{D}$ may be comparable in magnitude to $\Delta \Gamma$ (see e.g. [61]). In this case, agreement with the measured $x_{D}$ as the result of a cancellation between the SM and the new-physics contribution implies a mild fine-tuning around 10. In a more general phenomenological analysis, where flavour mixing is not necessarily aligned in the up-quark direction, one would have lesser effects in $D^{0}-\bar{D}^{0}$ as well as non-zero effects in down-type mixing observables. ${ }^{7}$ Such detailed flavour analysis is well beyond the scope of the present paper. For our purposes, the working hypotheses of an $\mathrm{SU}(2)_{h}$ horizontal plus an $\mathrm{SU}(3)_{\mathrm{HC}}$ symmetry need not be clearly ruled out by data.

\subsection{NGBs and baryon Lagrangian: the DM sector}

The $\mathrm{SU}(3)_{\mathrm{HC}}$-charged $\mathcal{F}_{L, R}$ fermions will form bound states. Interestingly for the DM phenomenology to follow, and as discussed in the Introduction, among these states, the lightest baryonic ones will be stable because of the accidentally conserved hyperbaryon

\footnotetext{
${ }^{7}$ These effects may be further tamed by increasing the $\mathrm{SU}(2)_{h}$-symmetry scale, at the price of predicting smaller discrepancies in $b \rightarrow s$ data [45].
} 
quantum number. Having fixed the dimension $N$ of the hypercolor space, $N=3$, the multiplicity of states is also fixed. It is easy to see that one obtains a quartet of spin- $3 / 2$ states, plus a doublet of spin- $1 / 2$ states, the latter being the lightest states. ${ }^{8}$ In analogy with the proton and the neutron of regular isospin, these two states will be

$$
\chi_{p} \sim \mathcal{F}_{2} \mathcal{F}_{2} \mathcal{F}_{3}, \quad \chi_{n} \sim \mathcal{F}_{2} \mathcal{F}_{3} \mathcal{F}_{3},
$$

where 2,3 label the flavor index. Scaling up the proton mass $m_{p}$ (see also discussion in section 4.1), we assume $m_{\chi}=m_{p} v_{\mathrm{HC}} / f_{\pi}$, with $f_{\pi}=93 \mathrm{MeV}$.

Let us now turn to the discussion of mesonic states. $\overline{\mathcal{F}} \mathcal{F}$ states are analogous to QCD's pions in the sense that they are the result of chiral $\mathrm{SU}(2)_{L}^{\mathcal{F}} \times \mathrm{SU}(2)_{R}^{\mathcal{F}}$ symmetry breaking at the scale $v_{\mathrm{HC}}$, while the pions are the result of $\mathrm{SU}(2)_{L} \times \mathrm{SU}(2)_{R}$ breaking at the scale $f_{\pi}$. Furthermore, the $\mathrm{SU}(2)_{L}$ appearing in two-flavor QCD and the $\mathrm{SU}(2)_{R}^{\mathcal{F}}$ appearing in the $\mathrm{HC}$ sector are both gauged. However, there is also an important difference between the QCD pions and the hyperpions. The $\mathrm{SU}(2)_{L}$ gauge symmetry is actually also broken by the Higgs vev at the scale $v=246 \mathrm{GeV}$. Both symmetry breakings generate NGBs, resulting in three Higgs NGBs and three pion NGBs. A linear combination of the Higgs NGBs and the pion NGBs forms the longitudinal polarizations of the $\mathrm{SU}(2)_{L}$ gauge bosons, while the orthogonal linear combinations constitute the physical pions. The latter become massive pNGBs due to the quark masses that are generated by the Higgs vev. Since the scales $v$ and $f_{\pi}$ are vastly different and the mixing angle $\alpha$ between Higgs NGBs and pion NGBs is given by the tiny $\tan \alpha=f_{\pi} / v \approx 4 \times 10^{-4}$, the longitudinal polarizations of the $\operatorname{SU}(2)_{L}$ gauge bosons are essentially the Higgs NGBs and the physical pions are essentially the pion NGBs. The situation is different in the $\mathrm{HC}$ sector, where the only symmetry breaking of the gauged $\mathrm{SU}(2)_{R}^{\mathcal{F}}=\mathrm{SU}(2)_{h}$ is the chiral symmetry breaking. Consequently, there are only the three hyperpion NGBs and they become exactly the longitudinal polarizations of the $\mathrm{SU}(2)_{h}$ gauge bosons.

We next discuss the Nambu-Goldstone boson (NGB) and baryon Lagrangian in detail. Our aim is to determine the $\chi_{p, n}$ interactions with SM matter, that are necessary for the DM phenomenology to follow. Apart from the differences mentioned above, the general structure of the Lagrangian that describes the interactions between the $\chi_{p, n}$ baryons, the $\overline{\mathcal{F}} \mathcal{F}$ mesons and the $\mathrm{SU}(2)_{h}$ bosons is largely analogous to the structure of the pion-nucleon Lagrangian [63, 64]. We will follow the line of argument and normalization conventions in ref. [65].

Given our chiral group $G^{\mathcal{F}} \equiv \mathrm{SU}(2)_{L}^{\mathcal{F}} \times \mathrm{SU}(2)_{R}^{\mathcal{F}}$, one introduces the field

$$
\Omega=\exp \left(i \frac{2 \Pi}{v_{\mathrm{HC}}}\right)
$$

with $\Pi$ the 'hyperpion' field $\Pi=\Pi^{a} T^{a}$, and $T^{a}=\tau^{a} / 2$ with the Pauli matrices $\tau^{a}$. Further denoting the elements of the global $\mathrm{SU}(2)_{L}^{\mathcal{F}}$ and $\mathrm{SU}(2)_{R}^{\mathcal{F}}$ symmetries as $L$ and $R$, one has

\footnotetext{
${ }^{8}$ Similarly as in Gell-Mann's 'eightfold way', such multiplets are obtained by first taking the fully symmetric irrep under spin $\times$ flavor, in our case $\mathrm{SU}(2 \times 2)$, which yields a $\mathbf{2 0}$, and then decomposing the latter into a direct sum of irreps under $\mathrm{SU}(2) \otimes \mathrm{SU}(2)$, which yields $(\mathbf{2}, \mathbf{2})+(\mathbf{4}, \mathbf{4})$.
} 
that $\Omega$ transforms as

$$
\Omega \rightarrow \Omega^{\prime}=R \Omega L^{\dagger} .
$$

The NGB Lagrangian at leading order in an expansion in derivatives is given by

$$
\mathcal{L}_{\mathrm{NGB}}=\frac{v_{\mathrm{HC}}^{2}}{4} \operatorname{tr}\left[\left(D_{\mu} \Omega\right)\left(D^{\mu} \Omega\right)^{\dagger}\right]
$$

where the covariant derivative reads

$$
D_{\mu} \Omega=\partial_{\mu} \Omega-i r_{\mu} \Omega+i \Omega l_{\mu}
$$

The currents $l_{\mu}$ and $r_{\mu}$ are in our case given by

$$
l_{\mu}=0, \quad r_{\mu}=-g_{H} H_{\mu}^{a} T^{a} .
$$

Consequently, the NGB Lagrangian can be written as

$$
\mathcal{L}_{\mathrm{NGB}}=\frac{v_{\mathrm{HC}}^{2}}{4} \operatorname{tr}\left[\partial_{\mu} \Omega \partial^{\mu} \Omega^{\dagger}\right]+\frac{1}{2} m_{H}^{2} H_{\mu}^{a} H^{a \mu}-i m_{H} v_{\mathrm{HC}} \operatorname{tr}\left[H_{\mu}\left(\partial^{\mu} \Omega\right) \Omega^{\dagger}\right],
$$

where the first term is the pure NGB Lagrangian, the second terms yields a mass for the vector bosons with $m_{H}=\frac{1}{2} g_{H} v_{\mathrm{HC}}$, and the third term leads to interactions between a vector boson and the NGBs. Using the definition of $\Omega$, one can expand

$$
\left(\partial^{\mu} \Omega\right) \Omega^{\dagger}=\frac{2 i}{v_{\mathrm{HC}}} \partial^{\mu} \Pi+\frac{1}{2 !}\left(\frac{2 i}{v_{\mathrm{HC}}}\right)^{2}\left[\Pi, \partial^{\mu} \Pi\right]+\frac{1}{3 !}\left(\frac{2 i}{v_{\mathrm{HC}}}\right)^{3}\left[\Pi,\left[\Pi, \partial^{\mu} \Pi\right]\right]+\ldots
$$

such that the third term in eq. (2.11) becomes

$$
\mathcal{L}_{\mathrm{NGB}} \supset m_{H} H_{\mu}^{a} \partial^{\mu} \Pi^{a}-\frac{g_{H}}{2} \varepsilon^{a b c} H_{\mu}^{a} \Pi^{b} \partial^{\mu} \Pi^{c}+\ldots .
$$

We observe that this leads to an inconvenient term that is linear in both $H_{\mu}^{a}$ and $\Pi^{a}$ and corresponds to a mixing between gauge bosons and NGBs. However, this term can be removed by a gauge transformation. To this end, we fix the gauge by adding the gaugefixing Lagrangian

$$
\mathcal{L}_{G F}=-\frac{1}{\xi_{H}} \operatorname{tr}\left[G_{H} G_{H}\right], \quad \text { where } \quad G_{H}=\partial^{\mu} H_{\mu}+\xi_{H} m_{H} \Pi,
$$

which results in

$$
\mathcal{L}_{G F}=-\frac{1}{2 \xi_{H}} \partial^{\mu} H_{\mu}^{a} \partial^{\mu} H_{\mu}^{a}-\frac{1}{2} \xi_{H} m_{H}^{2} \Pi^{a} \Pi^{a}-m_{H} H_{\mu}^{a} \partial^{\mu} \Pi^{a} .
$$

The first term yields the gauge-fixing condition for $H_{\mu}^{a}$, the second term is a $\xi_{H}$-dependent mass for the $\Pi^{a}$ fields, and the third term exactly cancels the unwanted mixing term in $\mathcal{L}_{\mathrm{NGB}}$.

We now turn to the matter field $\mathcal{X} \equiv\left(\chi_{p}, \chi_{n}\right)$. The latter transforms linearly as the fundamental irrep under the isospin subgroup $\mathrm{SU}(2)_{V}^{\mathcal{F}}$ with elements $V$. We can therefore write

$$
\mathcal{X} \rightarrow V \mathcal{X}
$$


Having defined our dynamical fields $\Omega$ and $\mathcal{X}$ and their chiral-symmetry transformation properties, we can now introduce the leading effective 'hyperbaryon' Lagrangian. First, we introduce the $\mathcal{X}$ covariant derivative

$$
i D_{\mu} \mathcal{X} \equiv\left(i \partial_{\mu}+i \Gamma_{\mu}+v_{\mu}^{(s)}\right) \mathcal{X}
$$

such that $D_{\mu} \mathcal{X}$ transforms like $\mathcal{X}$. Here

$$
i \Gamma_{\mu} \equiv \frac{i}{2}\left[\omega^{\dagger}\left(\partial_{\mu}-i r_{\mu}\right) \omega+\omega\left(\partial_{\mu}-i l_{\mu}\right) \omega^{\dagger}\right]
$$

where the external gauge currents $r_{\mu}$ and $l_{\mu}$ are given in eq. (2.10), and in our case $v_{\mu}^{(s)}=0$. The field $\omega$ is defined such that $\omega^{2}=\Omega$ and it transforms as

$$
\omega \rightarrow \omega^{\prime}=R \omega K^{-1}(L, R, \omega),
$$

with $K$ an $\mathrm{SU}(2)$-valued function of its arguments, in particular $\omega(x)$-dependent. In the case of an isospin transformation $R=L=V$ one has $K(V, V, \omega)=V$, namely the $\omega$ dependence drops. The most general Lagrangian describing pion-nucleon interactions with the smallest number of derivatives takes the form

$$
\mathcal{L}_{\Pi \mathcal{X}}=\overline{\mathcal{X}}\left(i \not D-m_{\chi}+g_{A} \gamma^{\mu} \gamma_{5} \frac{u_{\mu}}{2}\right) \mathcal{X}
$$

where $u_{\mu}$ is the so-called vielbein defined as

$$
\frac{u_{\mu}}{2} \equiv \frac{i}{2}\left[\omega^{\dagger}\left(\partial_{\mu}-i r_{\mu}\right) \omega-\omega\left(\partial_{\mu}-i l_{\mu}\right) \omega^{\dagger}\right] .
$$

The above Lagrangian generates interactions between $\chi$ and horizontal gauge bosons $H^{a}$ as well as $\overline{\mathcal{F}} \mathcal{F}$ mesons. Standard manipulations of the Lagrangian in eq. (2.20) lead to the following interaction terms including one or two boson fields

$$
\begin{aligned}
& \mathcal{L}_{\Pi \mathcal{X}} \supset \overline{\mathcal{X}}\left[-g_{H} \gamma^{\mu} H_{\mu}^{a}\left(T^{a}-\frac{1}{v_{\mathrm{HC}}} \varepsilon^{a b c} \Pi^{b} T^{c}\right)\left(\frac{1+g_{A} \gamma_{5}}{2}\right)\right. \\
&\left.+i g_{H} g_{A} \frac{m_{\chi}}{m_{H}} \gamma_{5} \Pi+\frac{1}{2 v_{\mathrm{HC}}^{2}} \gamma^{\mu} \varepsilon^{a b c}\left(\partial_{\mu} \Pi^{a}\right) \Pi^{b} T^{c}\right] \mathcal{X},
\end{aligned}
$$

where the parameters $m_{\chi}$ and $g_{A}$ are the chiral limits of the $\mathcal{X}$-multiplet mass and of the axial vector coupling of the multiplet to the hyperpions.

From eq. (2.22) we can read off all the $\chi \chi$ interactions that are necessary for the phenomenology to follow, in particular horizontal-boson exchange - relevant to DM direct detection - as well as one- or two-boson exchange - in principle relevant to the DM relic abundance. As concerns the latter case - discussed in detail in section 4.1 - the problem of estimating the relevant cross section resembles very closely the determination of baryonantibaryon annihilation close to threshold, for which we have abundant data. These data actually suggest that the $\chi \chi$-annihilation amplitude that one can estimate perturbatively from eq. (2.22) represents a sub-dominant contribution with respect to other contributions that cannot be captured with the $\mathcal{L}_{\Pi \mathcal{X}}$ Lagrangian, and that will be estimated directly from data. 


\section{Dark-Matter direct detection}

Both the DM multiplet and quarks interact with horizontal bosons. Therefore DM can scatter onto nuclei via the tree exchange of $\mathrm{SU}(2)_{h}$ bosons, and DM direct-detection (DD) data may in principle offer a separate probe of the $v_{\mathrm{HC}}$ scale.

The DM- $H_{\mu}^{a}$ interaction relevant for such process may be read off from eq. (2.22). As concerns (light) quark- $H_{\mu}^{a}$ interactions, also relevant for such process, the discussion in appendix A yields the following Lagrangian terms

$$
\begin{aligned}
\mathcal{L}_{q H}= & -g_{H}\left(\bar{u}_{L} U_{L}^{\dagger} \not H U_{L} u_{L}+\bar{d}_{L} D_{L}^{\dagger} \text { HH } D_{L} d_{L}\right. \\
& \left.+\bar{u}_{R} U_{R}^{\dagger} U_{R}^{\Psi^{u} u \dagger} \not H U_{R}^{\Psi^{u} u} U_{R} u_{R}+\bar{d}_{R} D_{R}^{\dagger} U_{R}^{\Psi^{d} d \dagger} \text { मH } U_{R}^{\Psi^{d} d} D_{R} d_{R}\right),
\end{aligned}
$$

where $u, d$ denote up- and down-type quark fields in the mass eigenbasis. For ease of notation, we will henceforth use the redefinitions

$$
\begin{array}{rlrl}
U_{L} & \rightarrow \mathcal{U}_{L}, & D_{L} & \rightarrow \mathcal{D}_{L}, \\
U_{R}^{\Psi^{u} u} U_{R} & \rightarrow \mathcal{U}_{R}, \quad U_{R}^{\Psi^{d} d} D_{R} \rightarrow \mathcal{D}_{R} .
\end{array}
$$

Since the momentum flowing through the $H$-boson propagator is way below its mass, one can write the following local interaction Lagrangian

$$
\begin{aligned}
& \mathcal{L}_{\text {eff }}= \\
& -\frac{g_{H}^{2}}{m_{H}^{2}}\left[\overline{\mathcal{X}} \gamma^{\mu} T^{a}\left(\frac{1+g_{A} \gamma_{5}}{2}\right) \mathcal{X}\right]\left[\left(\bar{u}_{L} \mathcal{U}_{L}^{\dagger} \gamma_{\mu} \mathcal{T}^{a} \mathcal{U}_{L} u_{L}+\{L \rightarrow R\}\right)+\{u \rightarrow d, \mathcal{U} \rightarrow \mathcal{D}\}\right],
\end{aligned}
$$

where in the first squared bracket $T^{a}$ denote the $\mathrm{SU}(2)_{h}$ generators, whereas in the second squared bracket $\mathcal{T}^{a}$ are $3 \times 3$ matrices defined by

$$
\mathcal{T}^{a} \equiv\left(\begin{array}{ll}
0 & \\
& T^{a}
\end{array}\right) .
$$

The quark bilinears in eq. (3.3) are to be evaluated between external nucleons. Under the assumption (whose validity we will discuss below) that both initial- and final-state nucleons are at rest, the required matrix elements may be parameterized as follows

$$
\begin{aligned}
&\left.\left\langle N\left(p^{\prime}\right)\left|\bar{q} \gamma^{\mu} q\right| N(p)\right\rangle\right|_{\vec{p}=\vec{p}^{\prime}=0}=F_{1}^{q / N}(0) \bar{u}_{N}\left(p^{\prime}\right) \gamma^{\mu} u_{N}(p), \\
&\left.\left\langle N\left(p^{\prime}\right)\left|\bar{q} \gamma^{\mu} \gamma_{5} q\right| N(p)\right\rangle\right|_{\vec{p}=\vec{p}^{\prime}=0}=F_{A}^{q / N}(0) \bar{u}_{N}\left(p^{\prime}\right) \gamma^{\mu} \gamma_{5} u_{N}(p) .
\end{aligned}
$$

The forward $F_{1, A}$ form factors can be parameterized as customary (see e.g. [66])

$$
\begin{aligned}
& F_{1}^{q / N}(0)=n_{q}^{N}, \\
& F_{A}^{q / N}(0)=\Delta q^{N},
\end{aligned}
$$

where $n_{q}^{N}$ counts the number of valence quarks $q$ within nucleon $N$ (e.g. $n_{s}^{p}=0$ ). Besides we use

$$
\Delta u^{p}=0.90, \quad \Delta d^{p}=-0.38, \quad \Delta s^{p}=-0.03,
$$

with $\Delta q^{n}$ obtained by isospin exchange. 
The DM $-N($ with $N=p, n)$ elastic cross section can then be written as

$$
\sigma^{N}=\frac{M_{N}^{2}}{\pi v_{\mathrm{HC}}^{4}}\left(\mathcal{V}_{N}+3 g_{A}^{2} \mathcal{A}_{N}\right)
$$

where the two terms on the r.h.s. are usually denoted as spin-independent (SI) and respectively spin-dependent (SD) in the literature. The constants $\mathcal{V}_{N}, \mathcal{A}_{N}$ are defined as

$$
\begin{aligned}
& \mathcal{V}_{N} \equiv \frac{1}{2} \sum_{a}\left|X_{V N}^{a}\right|^{2}, \\
& \mathcal{A}_{N} \equiv \frac{1}{2} \sum_{a}\left|X_{A N}^{a}\right|^{2},
\end{aligned}
$$

with

$$
\begin{aligned}
& X_{V N}^{a}=n_{u}^{N}\left(\bar{U}_{R}^{a}+\bar{U}_{L}^{a}\right)+n_{d}^{N}\left(\bar{D}_{R}^{a}+\bar{D}_{L}^{a}\right), \\
& X_{A N}^{a}=\Delta u^{N}\left(\bar{U}_{R}^{a}-\bar{U}_{L}^{a}\right)+\Delta d^{N}\left(\bar{D}_{R}^{a}-\bar{D}_{L}^{a}\right)+\Delta s^{N}\left(\bar{S}_{R}^{a}-\bar{S}_{L}^{a}\right),
\end{aligned}
$$

and

$$
\bar{U}_{L, R}^{a} \equiv\left(\mathcal{U}_{L, R}^{\dagger} \mathcal{T}^{a} \mathcal{U}_{L, R}\right)_{11}, \quad \bar{D}_{L, R}^{a} \equiv\left(\mathcal{D}_{L, R}^{\dagger} \mathcal{T}^{a} \mathcal{D}_{L, R}\right)_{11}, \quad \bar{S}_{L, R}^{a} \equiv\left(\mathcal{D}_{L, R}^{\dagger} \mathcal{T}^{a} \mathcal{D}_{L, R}\right)_{22}
$$

A numerical survey of eq. (3.8) yields

$$
\sigma^{N} \simeq 1.09\left(\frac{10 \mathrm{TeV}}{v_{\mathrm{HC}}}\right)^{4} 10^{-44} \mathrm{~cm}^{2} \times[0,3]
$$

where the last factor on the r.h.s. provides the range of values spanned by the $\mathcal{V}_{N}+3 g_{A}^{2} \mathcal{A}_{N}$ factor in eq. (3.8) as the $\mathcal{U}_{L, R}$ and $\mathcal{D}_{L, R}$ matrices are scanned over with the constraint $\mathcal{U}_{L}^{\dagger} \mathcal{D}_{L}=V_{\text {CKM }}$ (and no other constraint, in particular from flavor observables). ${ }^{9}$ We remark that, even for $\mathcal{V}_{N}+3 g_{A}^{2} \mathcal{A}_{N}$ as large as it gets, $\sigma^{N}$ remains safely below (a naive high-mass extrapolation of) the Xenon1T bound [67], keeping in mind that for $v_{\mathrm{HC}} \geq 10 \mathrm{TeV}$, one has $M_{\chi} \geq 100 \mathrm{TeV}$.

As well known, the analytic approach leading to eq. (3.8) has a number of limitations, that have been discussed in refs. [68-85]. Among them is the necessity to include renormalization-group running effects from the scale of the effective interaction between the DM and the quarks, down to the scale of the recoil energy that is measured in DM direct-detection experiments. This effect may be estimated by the DirectDM $[66,84,85]$ code. Another potentially important effect is the departure of eqs. (3.5) from the static limit, whereby one introduces form factors depending on $q^{2}$, with $q=p^{\prime}-p$. This effect may be quantified with the DMFormFactor [69-71] code.

\footnotetext{
${ }^{9}$ In this scan we took $g_{A}=1.27$, as in QCD. In doing so, we assume that QCD's $g_{A}$ at scales well below QCD's confinement scale should be similar to HC's $g_{A}$ at scales well below HC's confinement scale. This should be the case if $N_{\mathrm{HC}}=N_{c}$, if the non-perturbative dynamics is in both cases dominated by the two lightest (hyper)quarks, and if one can neglect (hyper)quark masses. We also note that $\mathcal{A}_{N} \ll \mathcal{V}_{N}$, hence $g_{A}$ does not need to be fixed very precisely.
} 
In our context, where the analytic procedure yields a direct-detection signal safely below experimental limits, the above effects would be relevant only if they enhanced the signal by orders of magnitude. Ref. [86] found that actually the numerical estimate obtained with the DirectDM plus DMFormFactor codes is lower with respect to the analytic result, by a factor not exceeding 2 for $M_{\chi} \lesssim 1.5 \mathrm{TeV}$ and for the interaction strength considered in that work. We extended that analysis to our case where the interaction strength is of order $1 / v_{\mathrm{HC}}^{2}$, with $v_{\mathrm{HC}}$ in the range in eq. (2.2), and where $M_{\chi}=10 v_{\mathrm{HC}}$ (see below eq. (2.5)). We find again that $\sigma_{\text {analytic }}^{N}>\sigma_{\text {numerical }}^{N}$, by a factor close to 4, i.e. even larger than in ref. [86]. This fact is not surprising, because even if the DM approaches the nucleus with a momentum of order $M_{\chi} v$, with $v \sim 10^{-3}$, the recoil energy does not increase indefinitely as $M_{\chi}$ increases, but instead it reaches the maximum value $M_{\mathcal{N}}(2 v)^{2} / 2$, with $M_{\mathcal{N}}$ the mass of the nucleus $\mathcal{N}$.

\section{Dark Matter relic density}

We wish to estimate the relic density of our DM multiplet $\mathcal{X}$ at present time, $\Omega_{0} h^{2}$. The first step towards this end is the determination of $\Omega h^{2}$ at the 'freeze-out' temperature $T_{f}$. In our case of a single, mass-degenerate, DM multiplet $\mathcal{X}, T_{f}$ is determined recursively from the 'matching' relation $[87,88]$

$$
x_{f}=\ln \frac{0.038 g_{\mathrm{eff}} M_{\mathrm{Pl}} m_{\chi}\left\langle\sigma_{\mathrm{eff}} v\right\rangle}{g_{*}^{1 / 2} x_{f}^{1 / 2}},
$$

where $x_{f}=m_{\chi} / T_{f}, g^{*}$ is the number of effectively relativistic d.o.f. at $T_{f}$ and $g_{\text {eff }}$ the number of internal d.o.f. of the $\mathcal{X}$, given in our case by

$$
g_{\text {eff }}=2 g_{p},
$$

and $g_{n}=g_{p}$ is the number of internal d.o.f. of the $\chi_{n}$ or $\chi_{p}$ particles. ${ }^{10}$

The main dynamical quantity, in eq. (4.1) as well as in the other steps towards determining $\Omega_{0} h^{2}$, is $\left\langle\sigma_{\text {eff }} v\right\rangle$. In our case of mass-degenerate $\chi_{i}[88]$

$$
\sigma_{\mathrm{eff}} \equiv \frac{1}{4} \sum_{i, j} \sigma_{i j}
$$

where $\sigma_{i j}$ is the annihilation cross section of $\bar{\chi}_{i}$ and $\chi_{j}$ and $i, j \in\{p, n\}$.

The second step towards obtaining $\Omega_{0} h^{2}$ is to estimate the post-freeze-out annihilation efficiency $J[88,89]$

$$
J \equiv \int_{x_{f}}^{\infty} \frac{\left\langle\sigma_{\text {eff }} v\right\rangle}{x^{2}} d x
$$

whence $\Omega_{0} h^{2}$ can be estimated as ${ }^{11}$

$$
\Omega_{0} h^{2}=\sqrt{\frac{45}{\pi}} \frac{s_{0}}{\rho_{c}} \frac{1}{g_{*}^{1 / 2} M_{\mathrm{Pl}} J} \simeq \frac{1.07 \times 10^{9} \mathrm{GeV}^{-1}}{g_{*}^{1 / 2} M_{\mathrm{Pl}} J} .
$$

\footnotetext{
${ }^{10}$ Remembering the definitions in eq. (2.5), $\chi_{p, n}$ although e.m.-neutral, cannot be Majorana fermions, hence $g_{p, n}=4$.

${ }^{11}$ One can derive this relation by using $H(T)=\sqrt{8 \pi^{3} g_{*} / 90} T^{2} / M_{\mathrm{Pl}}, s=2 \pi^{2} g_{*} T^{3} / 45$ and $\rho_{c}=$ $3 H_{0}^{2} /\left(8 \pi G_{N}\right)$, with $H\left(T_{0}\right) \equiv H_{0}=100 h \mathrm{~km} /(\mathrm{s} \mathrm{Mpc})$, as customary.
} 


\subsection{Calculation of $\left\langle\sigma_{\text {eff }} v\right\rangle$ and $J$}

For composite baryon-like objects like the $\chi$, the dominant processes contributing to $\bar{\chi} \chi$ annihilation take place in the strongly coupled regime such that it is not straightforward to calculate the cross section from first principles. In particular, as known from nucleonantinucleon annihilation (for reviews, see e.g. [90-93]), an important role is played by processes in which quarks and antiquarks inside the baryon and antibaryon are rearranged into mesons. In this case, even without an actual annihilation of the quarks, baryon and antibaryon are "annihilated". Other important processes are those in which some of the quarks and antiquarks actually annihilate via the strong interaction. Given that these processes happen in the strongly coupled regime, other weakly coupled interactions like QED in the case of nucleon-antinucleon annihilation or the $\mathrm{SU}(2)_{h}$ interactions in the case of $\bar{\chi} \chi$ annihilation play a subdominant role.

Since our strongly coupled sector resembles two-flavor QCD and the $\chi$ fields are very similar to proton and neutron in QCD, we estimate the $\bar{\chi} \chi$ annihilation cross section by scaling up experimental data on nucleon-antinucleon annihilation from the scale of the proton mass $m_{p}$ to the scale $m_{\chi}$ (a similar assumption was adopted in ref. [17]). Before doing so, it is worth pointing out the expected differences between the QCD and HC cases that are not related to the overall scale. These differences - that are sources of potential uncertainty — include the following:

- Nucleon-antinucleon annihilation data display a certain degree of isospin dependence, but we are unable to determine whether the latter is significant. In fact, in [94] neutron-antiproton annihilation is roughly a factor 0.75 smaller than protonantiproton annihilation. While this might be an effect mostly at low $p_{\text {lab }}$ due to electromagnetic attraction of $p$ and $\bar{p}$, we are not aware of any study that quantified such effect. On the other hand, according to [91] the differences between the protonantiproton and neutron-antiproton annihilation cross section are not significant. As detailed below, we perform our DM-phenomenology calculations with several different fits, collected in table 2, and use the spread of the predictions obtained as an estimation of this error.

- In nucleon-antinucleon annihilation, approximately $5 \%$ of the resulting mesons contain strange quarks (see e.g. [91] and references therein), which are not present in two-flavor QCD. Due to the absence of analogous annihilation channels in the case of $\bar{\chi} \chi$ annihilation, we should, in principle, lower the scaled-up cross section by $5 \%$, and add a $5 \%$ one-sided uncertainty accounting for our naive treatment. In practice, since the uncertainties inherent in our DM-phenomenology calculations, notably that of the relic abundance, are not smaller than $10 \%$ [88], we neglect the above error.

- The masses of NGB final-state mesons are the consequence of Lagrangian terms that explicitly break the global diagonal $\mathrm{SU}(2)_{V}^{(\mathcal{F})}$ (hyper)isospin symmetry. In QCD, isospin is explicitly broken by the quark masses, which leads to a ratio of the pion mass and decay constant $m_{\pi} / f_{\pi} \approx 1.35$. Then, in nucleon-antinucleon annihilations such effect distorts the zero-meson-mass result by terms that are expectedly of order 
$\left(m_{\pi} / m_{N}\right)^{2} \approx 2 \%$. In our setup, hyperisospin is broken by the gauging of $\mathrm{SU}(2)_{h}$ such that the hyperpions become the longitudinal polarizations of the horizontal gauge bosons. The ratio of their mass and the hyperpion decay constant $v_{\mathrm{HC}}$ is $m_{H} / v_{\mathrm{HC}}=g_{H} / 2$. Therefore, for large $g_{H}$ the effect might be similar in size to the one in QCD, while for small $g_{H}$ the effect might be smaller than in QCD. We conclude that this effect may also be neglected.

- The masses of non-NGB final-state mesons like the vector mesons are non-zero also in the chiral limit. Furthermore, for (hyper)quark masses that are small with respect to the confinement scale, the masses of these mesons are essentially independent of the (hyper)quark masses (see e.g. [95] for a lattice study of the mass decomposition). In our setup, the masses and couplings of the vector hypermesons can slightly be affected by an expected mixing with the $\mathrm{SU}(2)_{h}$ gauge bosons. However, we expect that this mixing does not change the overall cross section significantly. We therefore overlook this source of systematic difference.

In the following, we will consider different fits to the nucleon-antinucleon annihilation cross section, and scale them up from the nucleon to the $m_{\chi}$ mass scale. As we argued, the spread across the predictions obtained from the different fits may provide a reasonable 'envelope' for the theoretical uncertainty associated with the differences between QCD and our HC sector. Nucleon-antinucleon annihilation has been studied by many different experiments. Several groups provide fits to experimental data on the annihilation cross section $\sigma_{\text {ann }}$ using the parameterization

$$
\sigma_{\text {ann }}=\frac{1}{m_{p}^{2}}\left(A+B \frac{m_{p}}{p_{\text {lab }}}+C \frac{m_{p}^{2}}{p_{\text {lab }}^{2}}\right),
$$

where $p_{\text {lab }}$ is the momentum of the antinucleon in the rest frame of the nucleon and $m_{p}$ is the proton mass. Fit results are shown in table 2. In most fits, the coefficient $C$ is set to 0 . A non-zero $C$ can provide a slightly better fit at $p_{\text {lab }}$ around $75 \mathrm{MeV}$ [96], corresponding to $v \sim 10^{-1}$. More generally, data used in the fits in table 2 span the velocity range $[0.05$, $0.9]$, which includes the velocities $\sim 10^{-1}$ relevant to our freeze-out dynamics.

We assume that the $\bar{\chi}_{i} \chi_{j}$ annihilation cross section $\sigma_{i j}$ can be parameterized as in eq. (4.6) with $m_{p} \rightarrow m_{\chi}$, and that it is hyper-isospin independent, i.e. $\sigma_{i j}=\left.\sigma_{\text {ann }}\right|_{m_{p} \rightarrow m_{\chi}}$ ' which, using eq. (4.3), yields

$$
\sigma_{\text {eff }}=\left.\sigma_{\text {ann }}\right|_{m_{p} \rightarrow m_{\chi}} .
$$

In order to compute the thermally averaged cross section $\left\langle\sigma_{\text {eff }} v\right\rangle$ and the post-freeze-out annihilation efficiency $J$, an expansion at low velocity and large $x=m_{\chi} / T$ is commonly employed [100]. However, the low-velocity expansion does not converge for a cross section of the form as the one given in eq. (4.6). Therefore, in the following, we perform the thermal averaging without such an expansion. To this end, we consider the definition of $\left\langle\sigma_{\text {eff }} v\right\rangle$ in terms of the integral [101]

$$
\left\langle\sigma_{\mathrm{eff}} v\right\rangle=\frac{4 x}{K_{2}^{2}(x)} \int_{1}^{\infty} d y \sqrt{y}(y-1) K_{1}(2 x \sqrt{y}) \sigma_{\mathrm{eff}}(y),
$$




\begin{tabular}{|c|c|c|c|c|c|c|}
\hline Fit \# & Ref. & $N \bar{N}$ & $p_{\text {lab }}[\mathrm{GeV}]$ & $A$ & $B$ & $C$ \\
\hline \hline 1 & {$[94]$} & $p \bar{p}$ & {$[0.26,0.47]$} & 86 & 84 & 0 \\
2 & {$[97]$} & $p \bar{p}$ & {$[0.40,0.60]$} & $66.5 \pm 4.1$ & $77.1 \pm 2.2$ & 0 \\
3 & {$[98]$} & $p \bar{p}$ & {$[1.90,1.96]$} & $19.2 \pm 5.7$ & $98.1 \pm 3.1$ & 0 \\
\hline \hline 4 & {$[94,99]$} & $n \bar{p}$ & {$[0.26,0.47]$} & 63 & 63 & 0 \\
5 & {$[96]$} & $N \bar{n}$ & {$[0.05,0.40]$} & $150.4 \pm 6.8$ & $48.0 \pm 2.2$ & 0 \\
6 & {$[96]$} & $N \bar{n}$ & {$[0.05,0.40]$} & $199.9 \pm 10.6$ & $23.9 \pm 4.1$ & $2.5 \pm 0.4$ \\
\hline
\end{tabular}

Table 2. Fit parameters describing experimental data on nucleon-antinucleon annihilation parameterized as $\sigma_{\mathrm{ann}}=\frac{1}{m_{p}^{2}}\left(A+B \frac{m_{p}}{p_{\text {lab }}}+C \frac{m_{p}^{2}}{p_{\text {lab }}^{2}}\right)$, where $p_{\text {lab }}$ is the momentum of the antinucleon in the rest frame of the nucleon and $m_{p}$ is the proton mass. All dimensionful parameters in [94, 96-98] have been expressed in units of $m_{p}$ such that the coefficients $A, B$, and $C$ used here are dimensionless.

where $K_{\alpha}$ is the modified Bessel function of the second kind of order $\alpha$, and we introduce the dimensionless variable $y=s /\left(4 m_{\chi}^{2}\right)$ with the Mandelstam variable $s$ denoting the square of the $\bar{\chi} \chi$ center-of-mass energy. Changing variables in eq. (4.6) using $p_{\text {lab }}=2 m_{\chi} \sqrt{y^{2}-y}$, the integral (4.8) can be expressed as

$$
\left\langle\sigma_{\mathrm{eff}} v\right\rangle=\frac{A f_{0}(x)+B f_{-1}(x)+C f_{-2}(x)}{m_{\chi}^{2}}
$$

where the functions $f_{k}(x)$ can be given in terms of Meijer's $G$ functions and the subscript $k$ corresponds to the power of $p_{\text {lab }}$ in eq. (4.6). Since $\left\langle\sigma_{\text {eff }} v\right\rangle$ is evaluated only for $x \geq x_{f} \approx 30$, it is convenient to expand $f_{k}(x)$ in powers of $1 / x$, which yields

$$
f_{k}(x)=\frac{2^{2+k} \Gamma(2+k / 2)}{\sqrt{\pi}} x^{-(1+k) / 2}\left(1+\sum_{n=1}^{\infty} \frac{c_{n}(k)}{x^{n}}\right),
$$

where the first three coefficients $c_{n}(k)$ are given by ${ }^{12}$

$$
\begin{aligned}
& c_{1}(k)=-\frac{25}{16}+\frac{7}{4} k+\frac{5}{16} k^{2}, \\
& c_{2}(k)=\frac{1305}{512}-\frac{171}{64} k+\frac{343}{256} k^{2}+\frac{39}{64} k^{3}+\frac{25}{512} k^{4}, \\
& c_{3}(k)=-\frac{8115}{2048}+\frac{255}{256} k-\frac{6795}{1024} k^{2}-\frac{585}{256} k^{3}-\frac{375}{2048} k^{4} .
\end{aligned}
$$

Using these results, it is straightforward to determine the post-freeze-out annihilation efficiency $J$ from eq. (4.4). We express it as

$$
J=\frac{A g_{0}\left(x_{f}\right)+B g_{-1}\left(x_{f}\right)+C g_{-2}\left(x_{f}\right)}{m_{\chi}^{2}},
$$

where

$$
g_{k}\left(x_{f}\right)=\frac{2^{3+k} \Gamma(2+k / 2)}{\sqrt{\pi}(3+k)} x_{f}^{-(3+k) / 2}\left(1+\sum_{n=1}^{\infty} \frac{3+k}{3+k+2 n} \frac{c_{n}(k)}{x_{f}^{n}}\right) .
$$

\footnotetext{
${ }^{12}$ We verified that this series converges so quickly that terms beyond $c_{1}$ provide corrections way below $1 \%$.
} 

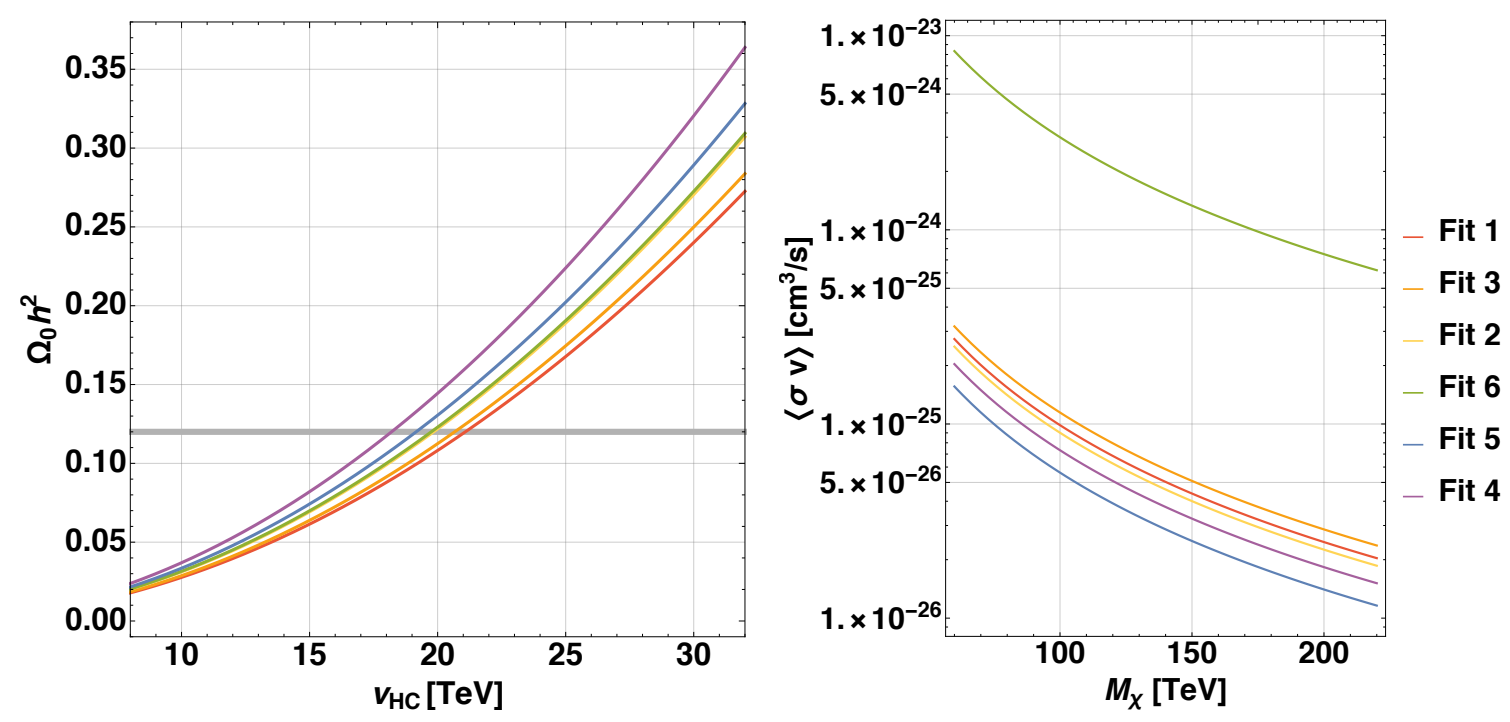

Figure 1. Left panel: present DM relic density as a function of $v_{\mathrm{HC}}$ for the different fits in table 2 (see text for details). The thick horizontal gray line represents the measured value $\Omega_{0} h^{2}=0.12$. Right panel: estimate (see text for details) of the velocity-averaged DM-DM annihilation cross section into anything else, $\left\langle\sigma_{\mathrm{ann}} v\right\rangle$, as a function of the DM mass, for the same fits as the left panel.

\section{$4.2 \Omega_{0} h^{2}$ prediction}

We can now use the thermally averaged cross section in eq. (4.9) to determine $\Omega_{0} h^{2}$ via eqs. (4.1), (4.4) and (4.5). It is interesting to note that ours is basically a one-parameter model, because the only quantity we can toggle is $v_{\mathrm{HC}}$ - within the range in eq. (2.2), with $m_{\chi} \simeq 10 v_{\mathrm{HC}}$, see below eq. (2.5). The $\Omega_{0} h^{2}$ prediction as a function of $v_{\mathrm{HC}}$ is presented in figure 1 (left panel). The different lines correspond to a $\chi \chi$ annihilation cross section estimated from the different fits in table 2. Each line should be attached a theory error of around $10 \%$ in the prediction of $\Omega_{0} h^{2}$, due to the analytic procedure we used to estimate the relic density [88]. This error is not reported in the figure to avoid clutter. Quite remarkably, the measured value $\Omega_{0} h^{2}=0.12$ is easily reproduced for $v_{\mathrm{HC}}$ in the range of eq. (2.2), for any of the fits in table 2. In particular, the $\Omega_{0} h^{2}$ constraint tends to select $v_{\mathrm{HC}} \approx 20 \mathrm{TeV}$ and the spread of actual values due to the different fits is no larger than about $15 \%$.

One further comment is in order. Since our model has naturally stable hyperbaryons, one could speculate on the possibility of producing a hyperbaryon asymmetry as the mechanism behind the observed Dark-Matter abundance. This would lead to an 'asymmetric Dark Matter' scenario (see [102] for a review, and refs. [4, 6, 103-111, 115-120] for pioneering work in this direction). ${ }^{13}$ While we cannot exclude this possibility, translating it into a prediction for $\Omega_{0}$ is a highly model-dependent question. In particular, such possibility would require the presence of hyperbaryon-number-violating interactions, ${ }^{14}$ hence

\footnotetext{
${ }^{13}$ In the context of our specific model, a possibly workable construction may be in the spirit of refs. [121-123].

${ }^{14}$ One simple example is the operator $\bar{L} \varphi \mathcal{X} / \Lambda_{\mathrm{HBV}}^{3}$, with $L \equiv\left(l_{2}, l_{3}\right)^{T}, \mathcal{X} \equiv\left(\chi_{p}, \chi_{n}\right)$ and $\varphi$ the Higgs field.
} 
to hyperbaryon decay at potentially too large a rate. We emphasize, on the other hand, that our scenario is an example of a baryon-DM scenario leading naturally to a fully calculable DM abundance, arising from well-understood freeze-out dynamics. We believe such calculability to be a merit of our setup.

\subsection{Comments on Dark-Matter indirect detection}

The annihilation cross section in eq. (4.6) may also be used to place conservative upper bounds on the distortions in the measured cosmic-ray fluxes that our DM candidate may give rise to when annihilating. Limits on the cross section $\sigma(\chi \chi \rightarrow X)$ for DM-pair annihilation to a given final state $X$ are usually given as $\langle\sigma(\chi \chi \rightarrow X) v\rangle$, see e.g. [124]. We have

$$
\langle\sigma(\chi \chi \rightarrow X) v\rangle \leq \sum_{X}\langle\sigma(\chi \chi \rightarrow X) v\rangle=\left\langle\sigma_{\mathrm{ann}} v\right\rangle \approx \sigma_{\mathrm{ann}} \times \bar{v},
$$

where in the last step we take $\bar{v} \sim 10^{-3}$ (see e.g. [125]). In figure 1 (right panel) we show the r.h.s. of eq. (4.14) as a function of the DM mass, where we set $p_{\text {lab }}=10^{-3} m_{\chi}$. We see that, with one exception, all the fits produce a velocity-averaged DM-DM annihilation (total) cross section below $3 \times 10^{-25} \mathrm{~cm}^{3} / \mathrm{s}$ for $m_{\chi}>70 \mathrm{TeV}$ (corresponding to $v_{\mathrm{HC}}>7 \mathrm{TeV}$ ). This cross section is safely below the bounds in e.g. refs. $[126,127]$ for the same DM mass, while for higher DM masses the bounds are expected to be weaker and the prediction smaller. The one exception is fit 6 , whose prediction is a factor between about 30 and 50 larger than the other fits'. However, none of the fits takes into account data for relative nucleon velocities as low as $10^{-3}$, which corresponds to $p_{\text {lab }}=1 \mathrm{MeV}$. In fit 6 , the lowest data points are given for a $[50,100] \mathrm{MeV}$ bin. More recent data is available from an experiment measuring $\bar{p}$-nucleus annihilation at $p_{\text {lab }}=15 \mathrm{MeV}$ [128]. Under the assumption $\sigma_{\text {ann }}^{N \bar{p}} \propto Z A^{1 / 3}$, valid at very low energies for the $\bar{p}$-nucleus interaction $[129,130]$, this data can be rescaled to the $p \bar{p}$ case. Doing so, one finds that fit 6 clearly overshoots this data already at $p_{\text {lab }}=15 \mathrm{MeV}$, indicating that it may give much larger values for even smaller momenta. Given also the conservative nature of our estimate via eq. (4.14), i.e. the actual annihilation cross section in a given channel can be orders of magnitudes smaller than our estimated total annihilation cross section, we may conclude that our model is on the safe side of indirect-detection bounds.

This discussion underlines the importance of obtaining nucleon-antinucleon data at $p_{\text {lab }}$ as small as $1 \mathrm{MeV}$, even though this might be very challenging. In fact, such data would provide very useful information for baryon-like DM scenarios.

\section{Conclusions}

We extend the SM by two flavors of massless vector-like fermions $\mathcal{F}$ charged under a new, 'hypercolor' (HC), strong force. An automatic outcome of this setup is the fact that the $\mathcal{F}$ fermions are endowed with an accidentally conserved hyperbaryon number, which makes the lightest hyperbaryons stable, and as such potential candidates for Dark Matter (DM).

The UV completions in appendix B do not generate such terms since they conserve hyperbaryon number. It may however be interesting to relate the scale $\Lambda_{\mathrm{HBV}}$ with the size of the DM asymmetry. 
We then gauge the $\mathrm{SU}(2)_{h}$ diagonal subgroup of two global $\mathrm{SU}(2)$ symmetries, one in the $\mathrm{HC}$ sector and the other one in the SM. The former is the chiral symmetry $\mathrm{SU}(2)_{R}^{\mathcal{F}}$ of righthanded hyperquarks, while the latter is the horizontal flavor symmetry of the two heavier generations of left-handed SM fermions. This gauging has several desired consequences:

- A mass term for the $\mathrm{SU}(2)_{h}$ gauge bosons is generated by the chiral symmetry breaking in the HC sector and the corresponding NGBs become the longitudinal polarizations of the $\mathrm{SU}(2)_{h}$ gauge bosons.

- The $\mathrm{SU}(2)_{h}$ gauge bosons couple to the SM fermions in such a way that they can address experimental hints for lepton universality violation in $b \rightarrow s$ data, as advocated in $[45]$.

- The $\mathrm{SU}(2)_{h}$ gauge bosons connect the baryon-like DM candidates in the HC sector to the SM fields, thereby playing the role of the DM mediator.

In order to generate the SM Higgs Yukawa couplings in the presence of the chiral SU(2) $h$ gauge group, additional fermions $\Psi$ are introduced. These fermions are vector-like under the SM gauge group but their right-handed components are charged under $\mathrm{SU}(2)_{h}$. They obtain masses from an 'extended hypercolor' mechanism as the $\mathcal{F}$ fermions condense, they mix with the right-handed SM fermions, and they have a Higgs Yukawa coupling together with the left-handed SM fermions. The final SM-like Higgs Yukawa couplings are then obtained from the mixing of right-handed SM fermions and $\Psi$ fermions. All masses of beyond-the-SM fields only depend on one single scale, the $\mathrm{SU}(2)_{h}$-symmetry breaking scale $v_{\mathrm{HC}}$, which is set by the $\mathcal{F}$-fermion condensate. It is expected to lie in the $[10,30] \mathrm{TeV}$ range by the mentioned $b \rightarrow s$ discrepancies.

We study the phenomenology of the hyperbaryon DM, in particular its relic density, expected to be produced by a mechanism of thermal freeze-out, and its direct-detection signals. Because our model has, basically, one single scale, our DM phenomenology predictions are functions of this one parameter. We find remarkable that the $v_{\mathrm{HC}}$ range mentioned above yields the correct relic density for the lightest $\mathcal{F}$ hyperbaryons. In particular, the relic-density constraint selects $v_{\mathrm{HC}} \approx 20 \mathrm{TeV}$, corresponding to a DM mass $m_{\chi} \simeq 10 v_{\mathrm{HC}} \approx$ $200 \mathrm{TeV}$. The stringent bounds from direct detection are quite comfortably satisfied. So our setup yields a composite DM candidate lying in the upper end of the mass range usually assumed for WIMP DM.

Taking advantage of nucleon-antinucleon annihilation data, we also provide a conservative estimate of indirect-detection signals of our scenario. In this context, we underline the importance of nucleon-antinucleon data at $p_{\text {lab }}$ as small as $1 \mathrm{MeV}$, as they would offer important insights on baryon-like DM scenarios.

In conclusion, within our setup the DM in the Universe is generated by an inherently flavorful mechanism, a horizontal symmetry, and the DM mass scale is unambiguously related to the horizontal symmetry breaking scale. Flavor discrepancies provide a two-sided bound for this scale, and quite interestingly this very range produces a DM phenomenology in accord with observations. Although our setup is inspired by the current anomalies in 
$b \rightarrow s$ data, ${ }^{15}$ the underlying idea may be easily adaptable to other collider discrepancies pointing to the tree-level exchange of a new gauge interaction with a symmetry-breaking scale in the tens-of- $\mathrm{TeV}$ range.

\section{Acknowledgments}

DG warmly thanks Eugenio Del Nobile, Filippo Sala and Pasquale Dario Serpico for discussions on DM direct- and indirect-detection dynamics. Exchanges with Véronique Bernard are also acknowledged. This work is supported by an ANR PRC (contract n. 202650) and by the Labex Enigmass.

\section{A Mixing between SM and VL fermions}

For each $f \in\{u, d, e\}$, the $2 \times 3$ matrices $\Delta_{f}$ in eq. (2.4) can be singular-value decomposed as

$$
\Delta_{f}=U_{L}^{\Delta_{f}^{\dagger}} \hat{\Delta}_{f} U_{R}^{\Delta_{f}}
$$

where $U_{L}^{\Delta_{f}}$ and $U_{R}^{\Delta_{f}}$ are $2 \times 2$ and $3 \times 3$ unitary matrices, respectively, and $\hat{\Delta}_{f}$ is a $2 \times 3$ rectangular diagonal matrix with two non-zero positive entries. Without loss of generality, one can perform the redefinition

$$
f_{R}^{\prime r} \rightarrow\left(U_{R}^{\Delta_{f} \dagger}\right)^{r k} f_{R}^{\prime k}, \quad y_{f}^{r} \rightarrow y_{f}^{k}\left(U_{R}^{\Delta_{f}}\right)^{k r}
$$

which entails that the mixing terms can be written as

$$
\mathcal{L} \supset-\Delta_{f}^{i j} \bar{\Psi}_{L}^{\prime f i} f_{R}^{\prime j}
$$

where now $i, j \in\{2,3\}$, i.e. with a slight abuse of notation, $\Delta_{f}$ can be restricted to be a $2 \times 2$ matrix given by

$$
\Delta_{f}=U_{L}^{\Delta_{f}^{\dagger}} \hat{\Delta}_{f}
$$

where $\hat{\Delta}_{f}$ is accordingly a $2 \times 2$ diagonal matrix.

Once the hyperquarks $\mathcal{F}$ form the condensate

$$
\left\langle\overline{\mathcal{F}}_{R}^{\alpha} \mathcal{F}_{L}^{j}\right\rangle=-\frac{1}{2} B_{\mathcal{F}} v_{\mathrm{HC}}^{2} \delta_{\alpha j} \approx-4 \pi v_{\mathrm{HC}}^{3} \delta_{\alpha j}
$$

where $\alpha$ is an index of the gauged $\mathrm{SU}(2)_{h}$ and $j$ is an index of the global $\mathrm{SU}(2)_{L}^{\mathcal{F}}$, the four-fermion operators in eq. (2.3) yield the mass terms

$$
-m_{\Psi}^{\prime i \alpha}\left(\bar{\Psi}_{L}^{i} \Psi_{R}^{\alpha}\right)+\text { h.c. }, \quad \text { where } \quad m_{\Psi}^{\prime i \alpha}=\frac{c_{i \alpha}+\tilde{c}_{i \alpha}}{2} B_{\mathcal{F}} \frac{v_{\mathrm{HC}}^{2}}{\Lambda_{\mathrm{EHC}}^{2}} .
$$

Consequently, for each $f \in\{u, d, e\}$ there is a $2 \times 4$ mass-mixing matrix

$$
M_{f}^{\prime}=\left(\Delta_{f} m_{\Psi^{f}}^{\prime}\right),
$$

\footnotetext{
${ }^{15}$ For other studies of a possible connection between DM and current $B$-physics discrepancies see [86, 131-159].
} 
such that

$$
\mathcal{L} \supset-\bar{\Psi}_{L}^{\prime f} M_{f}^{\prime}\left(\begin{array}{c}
f_{R}^{\prime} \\
\Psi_{R}^{\prime f}
\end{array}\right) .
$$

The singular value decomposition of the matrix $M_{f}^{\prime}$ yields

$$
M_{f}=\left(\begin{array}{ll}
0 m_{\Psi^{f}}
\end{array}\right)=U_{L}^{f \dagger} M_{f}^{\prime}\left(\begin{array}{cc}
U_{R}^{f^{\prime} f} & U_{R}^{f^{\prime} \Psi^{f}} \\
U_{R}^{\Psi^{\prime f} f} & U_{R}^{\Psi^{\prime f} \Psi^{f}}
\end{array}\right),
$$

where $U_{L}$ is a $2 \times 2$ unitary matrix, the $U_{R}$ are $2 \times 2$ submatrices of a $4 \times 4$ unitary matrix, and $m_{\Psi^{f}}$ is a $2 \times 2$ diagonal matrix with real positive entries. Using these unitary matrices to transform the fields to the mass eigenbasis (before EW symmetry breaking), the SM Yukawa matrices read

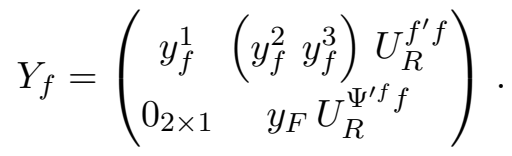

\section{B Possible UV completions}

The non-renormalizable four-fermion operators in eq. (2.3) are assumed to be generated from a (renormalizable) EHC sector. In this appendix, we demonstrate how such an EHC sector could be constructed and can serve as a consistent UV completion of the necessary non-renormalizable operators. To this end, we provide two examples:

- An extension in terms of a single heavy scalar field that, once integrated out, can generate both operators in eq. (2.3). This very minimal construction is analogous to bosonic technicolor [160].

- An extension of the gauge sector that results in new heavy vector bosons $X_{\mu}$ that generate the first operator in eq. (2.3). In this case, additional four-fermion operators are generated by the vector bosons accompanying the $X_{\mu}$ fields. Not all terms in eq. (2.4) are invariant under the extended gauge symmetry and are only generated once it is spontaneously broken. In order to generate the terms in the last line of eq. (2.4), new vector-like fermions are introduced.

The field content and possible renormalizable couplings in these two UV completions are described in the following. We note explicitly that both constructions preserve hyperbaryonnumber conservation.

\section{B.1 Scalar extension}

A new complex scalar field $\Phi$ with quantum numbers $(\mathbf{1}, \mathbf{2}, \mathbf{1}, \mathbf{1}, 0)$ under $\mathrm{SU}(3)_{\mathrm{HC}} \times$ $\mathrm{SU}(2)_{h} \times \mathrm{SU}(3)_{c} \times \mathrm{SU}(2)_{L} \times \mathrm{U}(1)_{Y}$ is introduced. ${ }^{16}$ This scalar has the Yukawa couplings

$$
\mathscr{L} \supset-\left(y_{\Phi F}^{j} \Phi^{\dagger}+\tilde{y}_{\Phi F}^{j} \Phi \epsilon_{h}\right) \overline{\mathcal{F}}_{L}^{j} \mathcal{F}_{R}-\left(y_{\Phi \Psi}^{i} \Phi^{\dagger}+\tilde{y}_{\Phi \Psi}^{i} \Phi \epsilon_{h}\right) \bar{\Psi}_{L}^{i} \Psi_{R}+\text { h.c. },
$$

\footnotetext{
${ }^{16}$ Our discussion focuses on the most straightforward viable example. Other, more complicated, scalar sectors may be possible for the same purpose. E.g., given that $\Phi$ has zero hypercharge, one may consider a pseudoreal scalar instead. Also, our main discussion focuses on a hypercolor-neutral $\Phi$, but one could likewise consider the possibility of complex scalars that are $\overline{\mathbf{3}}$ of $\mathrm{SU}(3)_{\mathrm{HC}}$.
} 
where $\epsilon_{h}$ is the anti-symmetric tensor of $\mathrm{SU}(2)_{h}$. At energy scales below the $\Phi$ mass $m_{\Phi}$, they lead to the effective four-fermion operators

$$
\frac{y_{\Phi \Psi}^{i} y_{\Phi F}^{* j}+\tilde{y}_{\Phi \Psi}^{i} \tilde{y}_{\Phi F}^{* j}}{m_{\Phi}^{2}}\left(\bar{\Psi}_{L}^{i} \Psi_{R}\right)\left(\overline{\mathcal{F}}_{R} \mathcal{F}_{L}^{j}\right)+\text { h.c. } .
$$

and

$$
\frac{\tilde{y}_{\Phi \Psi}^{i} y_{\Phi F}^{j}+y_{\Phi \Psi}^{i} \tilde{y}_{\Phi F}^{j}}{m_{\Phi}^{2}}\left(\bar{\Psi}_{L}^{i} \Psi_{R}\right) \epsilon_{h}\left(\overline{\mathcal{F}}_{L}^{j} \mathcal{F}_{R}\right)+\text { h.c. }
$$

\section{B.2 Gauge extension}

The $\Psi$ and $\mathcal{F}$ fields are replaced by the unified multiplets $\zeta_{L} \sim\left(\zeta_{L}^{\mathcal{F}}, \zeta_{L}^{u}, \zeta_{L}^{d}, \zeta_{L}^{e}\right)^{T}$ and $\zeta_{R} \sim\left(\zeta_{R}^{\mathcal{F}}, \zeta_{R}^{u}, \zeta_{R}^{d}, \zeta_{R}^{e}\right)^{T}$, which are both fundamental 10 representations of an SU(10) unified gauge group. In order to generate all necessary Yukawa couplings, also new vector-like fermions $\xi^{\prime u, d, e}$ are introduced. The full gauge symmetry in the UV is $\mathrm{SU}(10) \times \mathrm{SU}(2)_{h} \times$ $\mathrm{SU}(3)^{\prime} \times \mathrm{SU}(2)_{L} \times \mathrm{U}(1)^{\prime}$ with quantum numbers of all fermion and scalar fields shown in table 3 and table 4 , respectively. We note that the scalar sector in table 4 breaks $\mathrm{SU}(10) \times \mathrm{SU}(3)^{\prime} \times \mathrm{U}(1)^{\prime}$ to $\mathrm{SU}(3)_{\mathrm{HC}} \times \mathrm{SU}(3)_{c} \times \mathrm{U}(1)_{Y}$. This can be achieved by the subgroup embedding $\mathrm{SU}(10) \supset \mathrm{SU}(3)_{\mathrm{HC}} \times \mathrm{SU}(7)$, for which the fundamental $\mathrm{SU}(10)$ splits into a $(\mathbf{3}, \mathbf{1})$, corresponding to the hyperquarks $\mathcal{F}$, and a $(\mathbf{1}, \mathbf{7})$, corresponding to the unified multiplet $\left(\zeta^{u}, \zeta^{d}, \zeta^{e}\right)^{T}$. One further has $\mathrm{SU}(7) \supset \mathrm{SU}(3)_{7} \times \mathrm{U}(1)_{7}$, for which the 7 splits into $\left(\mathbf{3},+\frac{2}{3}\right) \oplus\left(\mathbf{3},-\frac{1}{3}\right) \oplus(\mathbf{1},-1)$, corresponding to $\zeta^{u}, \zeta^{d}$, and $\zeta^{e}$, respectively. With the above notation for the subgroups, the QCD and hypercharge gauge groups can be identified with the diagonal subgroups $\mathrm{SU}(3)_{c}=\left(\mathrm{SU}(3)_{7} \times \mathrm{SU}(3)^{\prime}\right)_{\text {diag }}$ and $\mathrm{U}(1)_{Y}=\left(\mathrm{U}(1)_{7} \times \mathrm{U}(1)^{\prime}\right)_{\text {diag }}$.

The fermion fields have the Yukawa couplings and mass terms

$$
\begin{aligned}
\mathscr{L} \supset & -\lambda_{u}^{i k} \bar{\zeta}_{L}^{i} \Phi_{u}^{\dagger} u_{R}^{\prime k}-\lambda_{d}^{i k} \bar{\zeta}_{L}^{i} \Phi_{d}^{\dagger} d_{R}^{\prime k}-\lambda_{e}^{i k} \bar{\zeta}_{L}^{i} \Phi_{e}^{\dagger} e_{R}^{\prime k} \\
& -\tilde{\lambda}_{u} \bar{\xi}_{L}^{\prime u} \Phi_{u} \zeta_{R}-\tilde{\lambda}_{d} \bar{\xi}_{L}^{\prime d} \Phi_{d} \zeta_{R}-\tilde{\lambda}_{e} \bar{\xi}_{L}^{\prime e} \Phi_{e} \zeta_{R} \\
& -\lambda_{U} \bar{Q}_{L}^{\prime} \tilde{\varphi} \xi_{R}^{\prime u}-\lambda_{D} \bar{Q}_{L}^{\prime} \varphi \xi_{R}^{\prime d}-\lambda_{E} \bar{L}_{L}^{\prime} \varphi \xi_{R}^{\prime e} \\
& -m_{\xi^{u}}^{\prime} \bar{\xi}_{L}^{\prime u} \xi_{R}^{\prime u}-m_{\xi^{d}}^{\prime} \bar{\xi}_{L}^{\prime d} \xi_{R}^{\prime d}-m_{\xi^{e}}^{\prime} \bar{\xi}_{L}^{\prime e} \xi_{R}^{\prime e} \\
& -y_{u}^{k} \bar{q}_{L}^{\prime 1} \tilde{\varphi} u_{R}^{\prime k}-y_{d}^{k} \bar{q}_{L}^{\prime 1} \varphi d_{R}^{\prime k}-y_{e}^{k} \bar{l}_{L}^{\prime 1} \varphi e_{R}^{\prime k},
\end{aligned}
$$

as well as their Hermitian conjugates. The last line is present also in the IR theory, while the first four lines generate the first and third line of eq. (2.4) at energy scales below the scalar vevs $\left\langle\Phi_{u, d, e}\right\rangle$. In particular, one finds

$$
\Delta_{u, d, e}^{i k}=\lambda_{u, d, e}^{i k}\left\langle\Phi_{u, d, e}\right\rangle, \quad y_{U, D, E}=\lambda_{U, D, E} \sin \theta_{u, d, e},
$$

and

$$
\begin{aligned}
\xi_{R}^{u, d, e} & =\xi_{R}^{\prime u, d, e} \cos \theta_{u, d, e}+\zeta_{R}^{u, d, e} \sin \theta_{u, d, e}, & \xi_{L}^{u, d, e} & =\xi_{L}^{u, d, e}, \\
\Psi_{R}^{\prime u, d, e} & =\zeta_{R}^{u, d, e} \cos \theta_{u, d, e}-\xi_{R}^{\prime u, d, e} \sin \theta_{u, d, e}, & \Psi_{L}^{\prime u, d, e} & =\zeta_{L}^{u, d, e}, \\
\mathcal{F}_{R} & =\zeta_{R}^{\mathcal{F}}, & \mathcal{F}_{L} & =\zeta_{L}^{\mathcal{F}},
\end{aligned}
$$

where the fermion mixing angles $\theta_{u, d, e}$ and the $\xi$ masses $m_{\xi u, d, e}$ are defined by

$$
\sin \theta_{u, d, e}=\frac{\tilde{\lambda}_{u, d, e}\left\langle\Phi_{u, d, e}\right\rangle}{m_{\xi^{u, d, e}}}, \quad m_{\xi^{u, d, e}}=\sqrt{\tilde{\lambda}_{u, d, e}^{2}\left\langle\Phi_{u, d, e}\right\rangle^{2}+m_{\xi^{u, d, e}}^{\prime 2}} .
$$




\begin{tabular}{|c|c|c|c|c|c|}
\hline Field & $\mathrm{SU}(10)$ & $\mathrm{SU}(2)_{h}$ & $\mathrm{SU}(3)^{\prime}$ & $\mathrm{SU}(2)_{L}$ & $\mathrm{U}(1)^{\prime}$ \\
\hline \hline$Q_{L}^{\prime}$ & $\mathbf{1}$ & $\mathbf{2}$ & $\mathbf{3}$ & $\mathbf{2}$ & $+1 / 6$ \\
$L_{L}^{\prime}$ & $\mathbf{1}$ & $\mathbf{2}$ & $\mathbf{1}$ & $\mathbf{2}$ & $-1 / 2$ \\
$q_{L}^{\prime 1}$ & $\mathbf{1}$ & $\mathbf{1}$ & $\mathbf{3}$ & $\mathbf{2}$ & $+1 / 6$ \\
$\ell_{L}^{\prime 1}$ & $\mathbf{1}$ & $\mathbf{1}$ & $\mathbf{1}$ & $\mathbf{2}$ & $-1 / 2$ \\
$u_{R}^{\prime 1,2,3}$ & $\mathbf{1}$ & $\mathbf{1}$ & $\mathbf{3}$ & $\mathbf{1}$ & $+2 / 3$ \\
$d_{R}^{\prime 1,2,3}$ & $\mathbf{1}$ & $\mathbf{1}$ & $\mathbf{3}$ & $\mathbf{1}$ & $-1 / 3$ \\
$e_{R}^{\prime 1,2,3}$ & $\mathbf{1}$ & $\mathbf{1}$ & $\mathbf{1}$ & $\mathbf{1}$ & -1 \\
\hline \hline$\xi_{L, R}^{\prime u}$ & $\mathbf{1}$ & $\mathbf{2}$ & $\mathbf{3}$ & $\mathbf{1}$ & $+2 / 3$ \\
$\xi_{L, R}^{\prime d}$ & $\mathbf{1}$ & $\mathbf{2}$ & $\mathbf{3}$ & $\mathbf{1}$ & $-1 / 3$ \\
$\xi_{L, R}^{\prime \prime}$ & $\mathbf{1}$ & $\mathbf{2}$ & $\mathbf{1}$ & $\mathbf{1}$ & -1 \\
\hline \hline$\zeta_{R}$ & $\mathbf{1 0}$ & $\mathbf{2}$ & $\mathbf{1}$ & $\mathbf{1}$ & 0 \\
$\zeta_{L}^{2,3}$ & $\mathbf{1 0}$ & $\mathbf{1}$ & $\mathbf{1}$ & $\mathbf{1}$ & 0 \\
\hline
\end{tabular}

Table 3. Quantum numbers of SM-like fermions (first block), new heavy vector-like fermions $\xi$ (second block), and unified fields $\zeta \sim\left(\zeta^{\mathcal{F}}, \zeta^{u}, \zeta^{d}, \zeta^{e}\right)^{T}$ (third block).

\begin{tabular}{|c|c|c|c|c|c|}
\hline Field & $\mathrm{SU}(10)$ & $\mathrm{SU}(2)_{h}$ & $\mathrm{SU}(3)^{\prime}$ & $\mathrm{SU}(2)_{L}$ & $\mathrm{U}(1)^{\prime}$ \\
\hline \hline$\varphi$ & $\mathbf{1}$ & $\mathbf{1}$ & $\mathbf{1}$ & $\mathbf{2}$ & $+1 / 2$ \\
\hline \hline$\Phi_{u}$ & $\overline{\mathbf{1 0}}$ & $\mathbf{1}$ & $\mathbf{3}$ & $\mathbf{1}$ & $+2 / 3$ \\
$\Phi_{d}$ & $\overline{\mathbf{1 0}}$ & $\mathbf{1}$ & $\mathbf{3}$ & $\mathbf{1}$ & $-1 / 3$ \\
$\Phi_{e}$ & $\overline{\mathbf{1 0}}$ & $\mathbf{1}$ & $\mathbf{1}$ & $\mathbf{1}$ & -1 \\
\hline
\end{tabular}

Table 4. Quantum numbers of SM-like Higgs scalar (first block) and new scalars (second block) that break $\mathrm{SU}(10) \times \mathrm{SU}(3)^{\prime} \times \mathrm{U}(1)^{\prime} \rightarrow \mathrm{SU}(3)_{c} \times \mathrm{U}(1)_{Y}$.

The heavy gauge bosons that receive their masses from the scalar vevs $\left\langle\Phi_{u, d, e}\right\rangle$ generate the four-fermion operators

$$
-\frac{2 \cos \theta_{u, d, e} V_{u, d, e}^{i j}}{\sqrt{\left\langle\Phi_{u}\right\rangle^{2}+\left\langle\Phi_{d}\right\rangle^{2}+\left\langle\Phi_{e}\right\rangle^{2}}}\left(\bar{\Psi}_{L}^{\prime i}{ }^{i u, d, e} \Psi_{R}^{\prime u, d, e}\right)\left(\overline{\mathcal{F}}_{R} \mathcal{F}_{L}^{j}\right)+\text { h.c. }
$$

where the matrices $V_{u, d, e}$ denote the $\mathrm{SU}(2)_{V}^{\mathcal{F}}$ isospin misalignment between the $\Psi_{L}^{\prime i u, d, e}$ and $\mathcal{F}_{L}^{j}$ fields.

Open Access. This article is distributed under the terms of the Creative Commons Attribution License (CC-BY 4.0), which permits any use, distribution and reproduction in any medium, provided the original author(s) and source are credited. 


\section{References}

[1] G. Bertone, D. Hooper and J. Silk, Particle dark matter: Evidence, candidates and constraints, Phys. Rept. 405 (2005) 279 [hep-ph/0404175] [INSPIRE].

[2] L. Roszkowski, E.M. Sessolo and S. Trojanowski, WIMP dark matter candidates and searches - current status and future prospects, Rept. Prog. Phys. 81 (2018) 066201 [arXiv: 1707.06277] [INSPIRE].

[3] Particle Data Group collaboration, Review of Particle Physics, Phys. Rev. D 98 (2018) 030001 [INSPIRE].

[4] S. Nussinov, Technocosmology: could a technibaryon excess provide a 'natural' missing mass candidate?, Phys. Lett. B 165 (1985) 55 [INSPIRE].

[5] R.S. Chivukula and T.P. Walker, Technicolor cosmology, Nucl. Phys. B 329 (1990) 445 [INSPIRE].

[6] S.M. Barr, R.S. Chivukula and E. Farhi, Electroweak Fermion Number Violation and the Production of Stable Particles in the Early Universe, Phys. Lett. B 241 (1990) 387 [INSPIRE].

[7] S.M. Barr, Baryogenesis, sphalerons and the cogeneration of dark matter, Phys. Rev. D 44 (1991) 3062 [INSPIRE].

[8] D.B. Kaplan, A Single explanation for both the baryon and dark matter densities, Phys. Rev. Lett. 68 (1992) 741 [INSPIRE].

[9] S.B. Gudnason, C. Kouvaris and F. Sannino, Dark Matter from new Technicolor Theories, Phys. Rev. D 74 (2006) 095008 [hep-ph/0608055] [INSPIRE].

[10] R. Foadi, M.T. Frandsen and F. Sannino, Technicolor Dark Matter, Phys. Rev. D 80 (2009) 037702 [arXiv: 0812.3406] [inSPIRE].

[11] M.Y. Khlopov and C. Kouvaris, Composite dark matter from a model with composite Higgs boson, Phys. Rev. D 78 (2008) 065040 [arXiv:0806.1191] [InSPIRE].

[12] T.A. Ryttov and F. Sannino, Ultra Minimal Technicolor and its Dark Matter TIMP, Phys. Rev. D 78 (2008) 115010 [arXiv:0809.0713] [InSPIRE].

[13] F. Sannino, Conformal Dynamics for TeV Physics and Cosmology, Acta Phys. Polon. B 40 (2009) 3533 [arXiv: 0911.0931] [INSPIRE].

[14] Y. Bai and R.J. Hill, Weakly Interacting Stable Pions, Phys. Rev. D 82 (2010) 111701 [arXiv: 1005.0008] [INSPIRE].

[15] M.R. Buckley and E.T. Neil, Thermal dark matter from a confining sector, Phys. Rev. D 87 (2013) 043510 [arXiv: 1209.6054] [INSPIRE].

[16] J.M. Cline, Z. Liu, G. Moore and W. Xue, Composite strongly interacting dark matter, Phys. Rev. D 90 (2014) 015023 [arXiv:1312.3325] [InSPIRE].

[17] O. Antipin, M. Redi and A. Strumia, Dynamical generation of the weak and Dark Matter scales from strong interactions, JHEP 01 (2015) 157 [arXiv: 1410.1817] [INSPIRE].

[18] O. Antipin, M. Redi, A. Strumia and E. Vigiani, Accidental Composite Dark Matter, JHEP 07 (2015) 039 [arXiv: 1503.08749] [INSPIRE].

[19] A. Mitridate, M. Redi, J. Smirnov and A. Strumia, Dark Matter as a weakly coupled Dark Baryon, JHEP 10 (2017) 210 [arXiv:1707.05380] [INSPIRE]. 
[20] Y. Bai, A.J. Long and S. Lu, Dark Quark Nuggets, Phys. Rev. D 99 (2019) 055047 [arXiv: 1810.04360] [INSPIRE].

[21] G.R. Farrar, A precision test of the nature of Dark Matter and a probe of the QCD phase transition, arXiv: 1805.03723 [INSPIRE].

[22] C. Gross, A. Polosa, A. Strumia, A. Urbano and W. Xue, Dark Matter in the Standard Model?, Phys. Rev. D 98 (2018) 063005 [arXiv:1803.10242] [InSPIRE].

[23] H. Beauchesne, E. Bertuzzo and G. Grilli Di Cortona, Dark matter in Hidden Valley models with stable and unstable light dark mesons, JHEP 04 (2019) 118 [arXiv:1809.10152] [INSPIRE].

[24] H. Beauchesne and G. Grilli di Cortona, Classification of dark pion multiplets as dark matter candidates and collider phenomenology, JHEP 02 (2020) 196 [arXiv:1910.10724] [INSPIRE].

[25] E. Hall, T. Konstandin, R. McGehee and H. Murayama, Asymmetric Matters from a Dark First-Order Phase Transition, arXiv:1911.12342 [INSPIRE].

[26] G.R. Farrar, Z. Wang and X. Xu, Dark Matter Particle in QCD, arXiv:2007.10378 [INSPIRE].

[27] G.D. Kribs and E.T. Neil, Review of strongly-coupled composite dark matter models and lattice simulations, Int. J. Mod. Phys. A 31 (2016) 1643004 [arXiv:1604.04627] [INSPIRE].

[28] S. Weinberg, Approximate symmetries and pseudoGoldstone bosons, Phys. Rev. Lett. 29 (1972) 1698 [INSPIRE].

[29] K. Griest and M. Kamionkowski, Unitarity Limits on the Mass and Radius of Dark Matter Particles, Phys. Rev. Lett. 64 (1990) 615 [InSPIRE].

[30] LHCb collaboration, Test of lepton universality with $B^{0} \rightarrow K^{* 0} \ell^{+} \ell^{-}$decays, JHEP 08 (2017) 055 [arXiv: 1705. 05802] [INSPIRE].

[31] LHCb collaboration, Search for lepton-universality violation in $B^{+} \rightarrow K^{+} \ell^{+} \ell^{-}$decays, Phys. Rev. Lett. 122 (2019) 191801 [arXiv:1903.09252] [INSPIRE].

[32] Belle collaboration, Test of lepton flavor universality in $B \rightarrow K^{*} \ell^{+} \ell^{-}$decays at Belle, arXiv: 1904.02440 [INSPIRE].

[33] Belle collaboration, Test of lepton flavor universality in $B \rightarrow \mathrm{K}^{+} \ell^{-}$decays, arXiv: 1908.01848 [INSPIRE].

[34] L. Di Luzio and M. Nardecchia, What is the scale of new physics behind the B-flavour anomalies?, Eur. Phys. J. C 77 (2017) 536 [arXiv:1706.01868] [INSPIRE].

[35] J. Aebischer, W. Altmannshofer, D. Guadagnoli, M. Reboud, P. Stangl and D.M. Straub, B-decay discrepancies after Moriond 2019, Eur. Phys. J. C 80 (2020) 252 [arXiv: 1903.10434] [INSPIRE].

[36] M. Algueró et al., Emerging patterns of New Physics with and without Lepton Flavour Universal contributions, Eur. Phys. J. C 79 (2019) 714 [Addendum ibid. 80 (2020) 511] [arXiv:1903.09578] [INSPIRE].

[37] M. Ciuchini et al., New Physics in $b \rightarrow s \ell^{+} \ell^{-}$confronts new data on Lepton Universality, Eur. Phys. J. C 79 (2019) 719 [arXiv:1903.09632] [InSPIRE].

[38] A. Datta, J. Kumar and D. London, The $B$ anomalies and new physics in $b \rightarrow s e^{+} e^{-}$, Phys. Lett. B 797 (2019) 134858 [arXiv:1903.10086]. 
[39] K. Kowalska, D. Kumar and E.M. Sessolo, Implications for new physics in $b \rightarrow s \mu \mu$ transitions after recent measurements by Belle and LHCb, Eur. Phys. J. C 79 (2019) 840 [arXiv: 1903.10932] [INSPIRE].

[40] A. Arbey, T. Hurth, F. Mahmoudi, D.M. Santos and S. Neshatpour, Update on the $b \rightarrow s$ anomalies, Phys. Rev. D 100 (2019) 015045 [arXiv:1904.08399] [INSPIRE].

[41] J. Fuentes-Martín and P. Stangl, Third-family quark-lepton unification with a fundamental composite Higgs, Phys. Lett. B 811 (2020) 135953 [arXiv:2004.11376] [INSPIRE].

[42] Z. Berezhiani, Unified picture of the particle and sparticle masses in SUSY GUT, Phys. Lett. B 417 (1998) 287 [hep-ph/9609342] [INSPIRE].

[43] B. Belfatto and Z. Berezhiani, How light the lepton flavor changing gauge bosons can be, Eur. Phys. J. C 79 (2019) 202 [arXiv:1812.05414] [INSPIRE].

[44] L. Di Luzio, M. Kirk, A. Lenz and T. Rauh, $\Delta M_{s}$ theory precision confronts flavour anomalies, JHEP 12 (2019) 009 [arXiv: 1909.11087] [INSPIRE].

[45] D. Guadagnoli, M. Reboud and O. Sumensari, A gauged horizontal SU(2) symmetry and $R_{K^{(*)}}$, JHEP 11 (2018) 163 [arXiv: 1807.03285] [INSPIRE].

[46] C. Kilic, T. Okui and R. Sundrum, Vectorlike Confinement at the LHC, JHEP 02 (2010) 018 [arXiv:0906.0577] [INSPIRE].

[47] R. Pasechnik, V. Beylin, V. Kuksa and G. Vereshkov, Chiral-symmetric technicolor with standard model Higgs boson, Phys. Rev. D 88 (2013) 075009 [arXiv:1304.2081] [INSPIRE].

[48] P. Lebiedowicz, R. Pasechnik and A. Szczurek, Search for technipions in exclusive production of diphotons with large invariant masses at the LHC, Nucl. Phys. B $\mathbf{8 8 1}$ (2014) 288 [arXiv:1309.7300] [INSPIRE].

[49] R. Pasechnik, V. Beylin, V. Kuksa and G. Vereshkov, Composite scalar Dark Matter from vector-like SU(2) confinement, Int. J. Mod. Phys. A 31 (2016) 1650036 [arXiv:1407.2392] [INSPIRE].

[50] S. Matsuzaki, K. Nishiwaki and R. Watanabe, Phenomenology of flavorful composite vector bosons in light of B anomalies, JHEP 08 (2017) 145 [arXiv:1706.01463] [INSPIRE].

[51] Z.G. Berezhiani and J.L. Chkareuli, Mass of the $t$ quark and the number of quark lepton generations, JETP Lett. 35 (1982) 612 [Erratum ibid. 36 (1982) 380] [Erratum ibid. 36 (1982) 312] [INSPIRE].

[52] Z.G. Berezhiani and J.L. Chkareuli, Quark-leptonic families in a model with $\mathrm{SU}(5) \times \mathrm{SU}(3)$ symmetry (in Russian), Sov. J. Nucl. Phys. 37 (1983) 618 [inSPIRE].

[53] Z.G. Berezhiani, The Weak Mixing Angles in Gauge Models with Horizontal Symmetry: A New Approach to Quark and Lepton Masses, Phys. Lett. B 129 (1983) 99 [INSPIRE].

[54] Z.G. Berezhiani, Horizontal Symmetry and Quark-Lepton Mass Spectrum: The $\mathrm{SU}(5) \times \mathrm{SU}(3)-h$ Model, Phys. Lett. B 150 (1985) 177 [INSPIRE].

[55] E. Witten, An SU(2) Anomaly, Phys. Lett. B 117 (1982) 324 [inSPIRE].

[56] S. Dimopoulos and L. Susskind, Mass Without Scalars, [INSPIRE].

[57] E. Eichten and K.D. Lane, Dynamical Breaking of Weak Interaction Symmetries, Phys. Lett. B 90 (1980) 125 [INSPIRE]. 
[58] Z.G. Berezhiani and J.L. Chkareuli, Low-energy horizontal symmetry of $\mathrm{SU}(3)_{H} \times \mathrm{U}(1)_{H}$ and $B \bar{B}$ oscillation (in Russian), Sov. J. Nucl. Phys. 52 (1990) 383 [inSPIRE].

[59] HFLAV collaboration, Averages of b-hadron, c-hadron, and $\tau$-lepton properties as of 2018, arXiv: 1909.12524 [INSPIRE].

[60] A.A. Petrov, Long-distance effects in charm mixing, in Proceedings of 6th International Workshop on Charm Physics, Manchester U.K. (2013) [arXiv: 1312.5304] [INSPIRE].

[61] A.F. Falk, Y. Grossman, Z. Ligeti, Y. Nir and A.A. Petrov, The $D^{0}-\bar{D}^{0}$ mass difference from a dispersion relation, Phys. Rev. D 69 (2004) 114021 [hep-ph/0402204] [INSPIRE].

[62] B. Belfatto, R. Beradze and Z. Berezhiani, The CKM unitarity problem: A trace of new physics at the TeV scale?, Eur. Phys. J. C 80 (2020) 149 [arXiv:1906.02714] [InSPIRE].

[63] H. Georgi, Weak Interactions and Modern Particle Theory, Dover Publications, Mineola U.S.A. (2009).

[64] J. Gasser, M.E. Sainio and A. Svarc, Nucleons with Chiral Loops, Nucl. Phys. B 307 (1988) 779 [INSPIRE].

[65] S. Scherer, Chiral Perturbation Theory: Introduction and Recent Results in the One-Nucleon Sector, Prog. Part. Nucl. Phys. 64 (2010) 1 [arXiv:0908.3425] [inSPIRE].

[66] F. Bishara, J. Brod, B. Grinstein and J. Zupan, DirectDM: a tool for dark matter direct detection, arXiv: 1708.02678 [INSPIRE].

[67] XENON collaboration, Dark Matter Search Results from a One Ton-Year Exposure of XENON1T, Phys. Rev. Lett. 121 (2018) 111302 [arXiv:1805.12562] [INSPIRE].

[68] J. Fan, M. Reece and L.-T. Wang, Non-relativistic effective theory of dark matter direct detection, JCAP 11 (2010) 042 [arXiv: 1008.1591] [INSPIRE].

[69] A.L. Fitzpatrick, W. Haxton, E. Katz, N. Lubbers and Y. Xu, The Effective Field Theory of Dark Matter Direct Detection, JCAP 02 (2013) 004 [arXiv:1203.3542] [INSPIRE].

[70] A.L. Fitzpatrick, W. Haxton, E. Katz, N. Lubbers and Y. Xu, Model Independent Direct Detection Analyses, arXiv:1211.2818 [INSPIRE].

[71] N. Anand, A.L. Fitzpatrick and W.C. Haxton, Weakly interacting massive particle-nucleus elastic scattering response, Phys. Rev. C 89 (2014) 065501 [arXiv:1308.6288] [INSPIRE].

[72] V. Cirigliano, M.L. Graesser and G. Ovanesyan, WIMP-nucleus scattering in chiral effective theory, JHEP 10 (2012) 025 [arXiv: 1205.2695] [INSPIRE].

[73] M. Cirelli, E. Del Nobile and P. Panci, Tools for model-independent bounds in direct dark matter searches, JCAP 10 (2013) 019 [arXiv:1307.5955] [INSPIRE].

[74] G. Barello, S. Chang and C.A. Newby, A Model Independent Approach to Inelastic Dark Matter Scattering, Phys. Rev. D 90 (2014) 094027 [arXiv: 1409.0536] [INSPIRE].

[75] R.J. Hill and M.P. Solon, Standard Model anatomy of WIMP dark matter direct detection II: QCD analysis and hadronic matrix elements, Phys. Rev. D 91 (2015) 043505 [arXiv:1409.8290] [INSPIRE].

[76] M. Hoferichter, P. Klos and A. Schwenk, Chiral power counting of one- and two-body currents in direct detection of dark matter, Phys. Lett. B $\mathbf{7 4 6}$ (2015) 410 [arXiv: 1503.04811] [INSPIRE]. 
[77] R. Catena and P. Gondolo, Global fits of the dark matter-nucleon effective interactions, JCAP 09 (2014) 045 [arXiv: 1405.2637] [INSPIRE].

[78] R.J. Hill and M.P. Solon, WIMP-nucleon scattering with heavy WIMP effective theory, Phys. Rev. Lett. 112 (2014) 211602 [arXiv:1309.4092] [InSPIRE].

[79] R.J. Hill and M.P. Solon, Universal behavior in the scattering of heavy, weakly interacting dark matter on nuclear targets, Phys. Lett. B 707 (2012) 539 [arXiv:1111.0016] [InSPIRE].

[80] M. Hoferichter, P. Klos, J. Menéndez and A. Schwenk, Analysis strategies for general spin-independent WIMP-nucleus scattering, Phys. Rev. D 94 (2016) 063505 [arXiv: 1605.08043] [INSPIRE].

[81] A. Kurylov and M. Kamionkowski, Generalized analysis of weakly interacting massive particle searches, Phys. Rev. D 69 (2004) 063503 [hep-ph/0307185] [INSPIRE].

[82] M. Pospelov and T. ter Veldhuis, Direct and indirect limits on the electromagnetic form-factors of WIMPs, Phys. Lett. B 480 (2000) 181 [hep-ph/0003010] [INSPIRE].

[83] J. Bagnasco, M. Dine and S.D. Thomas, Detecting technibaryon dark matter, Phys. Lett. B 320 (1994) 99 [hep-ph/9310290] [InSPIRE].

[84] F. Bishara, J. Brod, B. Grinstein and J. Zupan, Chiral Effective Theory of Dark Matter Direct Detection, JCAP 02 (2017) 009 [arXiv:1611.00368] [INSPIRE].

[85] F. Bishara, J. Brod, B. Grinstein and J. Zupan, From quarks to nucleons in dark matter direct detection, JHEP 11 (2017) 059 [arXiv:1707.06998] [INSPIRE].

[86] D. Guadagnoli, M. Reboud and P. Stangl, The Dark Side of 4321, JHEP 10 (2020) 084 [arXiv: 2005.10117] [INSPIRE].

[87] E.W. Kolb and M.S. Turner, The Early Universe, Front. Phys. 69 (1990) 1.

[88] K. Griest and D. Seckel, Three exceptions in the calculation of relic abundances, Phys. Rev. D 43 (1991) 3191 [INSPIRE].

[89] G. Jungman, M. Kamionkowski and K. Griest, Supersymmetric dark matter, Phys. Rept. 267 (1996) 195 [hep-ph/9506380] [INSPIRE].

[90] A.M. Green and J.A. Niskanen, Low-energy Anti-proton Physics in the Early LEAR Era, Prog. Part. Nucl. Phys. 18 (1987) 93 [InSPIRE].

[91] C.B. Dover, T. Gutsche, M. Maruyama and A. Faessler, The Physics of nucleon-anti-nucleon annihilation, Prog. Part. Nucl. Phys. 29 (1992) 87 [inSPIRE].

[92] C. Amsler, Nucleon-antinucleon annihilation at LEAR, 8, 2019 [arXiv:1908.08455] [INSPIRE].

[93] J.-M. Richard, Antiproton physics, Front. Phys. 8 (2020) 6 [arXiv:1912.07385].

[94] T. Kalogeropoulos and G.S. Tzanakos, Total and partial $\bar{p} d$ cross-sections from 0.26 to $0.47 \mathrm{GEV} / \mathrm{c}$, Phys. Rev. D 22 (1980) 2585 [InSPIRE].

[95] Y.-B. Yang et al., Meson Mass Decomposition from Lattice QCD, Phys. Rev. D 91 (2015) 074516 [arXiv: 1405.4440] [INSPIRE].

[96] M. Astrua et al., Antineutron nucleus annihilation cross-sections below 400-MeV/c, Nucl. Phys. A 697 (2002) 209 [inSPIRE]. 
[97] W. Brückner et al., Search for a Narrow Resonance in Anti-proton - Proton Annihilation Cross-sections in the Beam Momentum Range Between 400-MeV/c and 600-MeV/c, Phys. Lett. B 197 (1987) 463 [Erratum ibid. 199 (1987) 596] [INSPIRE].

[98] D.I. Lowenstein et al., Search for narrow structure in proton-antiproton annihilation cross sections from 1900 to $1960 \mathrm{MeV}$, AIP Conf. Proc. 132 (1985) 137 [InSPIRE].

[99] R. Bizzarri, P. Guidoni, F. Marcelja, F. Marzano, E. Castelli and M. Sessa, Anti-proton-deuteron low-energy cross-section, Nuovo Cim. A 22 (1974) 225 [INSPIRE].

[100] M. Srednicki, R. Watkins and K.A. Olive, Calculations of Relic Densities in the Early Universe, Nucl. Phys. B 310 (1988) 693 [InSPIRE].

[101] M. Cannoni, Relativistic $\left\langle\sigma v_{\text {rel }}\right\rangle$ in the calculation of relics abundances: a closer look, Phys. Rev. D 89 (2014) 103533 [arXiv:1311.4494] [INSPIRE].

[102] K. Petraki and R.R. Volkas, Review of asymmetric dark matter, Int. J. Mod. Phys. A 28 (2013) 1330028 [arXiv:1305.4939] [INSPIRE].

[103] R. Foot, H. Lew and R.R. Volkas, A Model with fundamental improper space-time symmetries, Phys. Lett. B 272 (1991) 67 [InSPIRE].

[104] R. Foot, H. Lew and R.R. Volkas, Possible consequences of parity conservation, Mod. Phys. Lett. A 7 (1992) 2567 [INSPIRE].

[105] R. Foot and R.R. Volkas, Neutrino physics and the mirror world: How exact parity symmetry explains the solar neutrino deficit, the atmospheric neutrino anomaly and the LSND experiment, Phys. Rev. D 52 (1995) 6595 [hep-ph/9505359] [INSPIRE].

[106] T.D. Lee and C.-N. Yang, Question of Parity Conservation in Weak Interactions, Phys. Rev. 104 (1956) 254 [INSPIRE].

[107] I.Y. Kobzarev, L.B. Okun and I.Y. Pomeranchuk, On the possibility of experimental observation of mirror particles, Sov. J. Nucl. Phys. 3 (1966) 837 [InSPIRE].

[108] M. Pavsic, External inversion, internal inversion, and reflection invariance, Int. J. Theor. Phys. 9 (1974) 229 [hep-ph/0105344] [INSPIRE].

[109] S.I. Blinnikov and M.Y. Khlopov, On possible effects of 'mirror' particles, Sov. J. Nucl. Phys. 36 (1982) 472 [INSPIRE].

[110] Z. Berezhiani, D. Comelli and F.L. Villante, The Early mirror universe: Inflation, baryogenesis, nucleosynthesis and dark matter, Phys. Lett. B 503 (2001) 362 [hep-ph/0008105] [INSPIRE].

[111] A.Y. Ignatiev and R.R. Volkas, Mirror dark matter and large scale structure, Phys. Rev. D 68 (2003) 023518 [hep-ph/0304260] [INSPIRE].

[112] L. Bento and Z. Berezhiani, Leptogenesis via collisions: The Lepton number leaking to the hidden sector, Phys. Rev. Lett. 87 (2001) 231304 [hep-ph/0107281] [INSPIRE].

[113] L. Bento and Z. Berezhiani, Baryon asymmetry, dark matter and the hidden sector, Fortsch. Phys. 50 (2002) 489 [INSPIRE].

[114] Z. Berezhiani, Mirror world and its cosmological consequences, Int. J. Mod. Phys. A 19 (2004) 3775 [hep-ph/0312335] [INSPIRE].

[115] R. Foot and R.R. Volkas, Was ordinary matter synthesized from mirror matter? An Attempt to explain why Omega(Baryon) approximately equal to 0.2 Omega(Dark), Phys. Rev. D 68 (2003) 021304 [hep-ph/0304261] [INSPIRE]. 
[116] R. Foot and R.R. Volkas, Explaining Omega(Baryon) approximately 0.2 Omega(Dark) through the synthesis of ordinary matter from mirror matter: A More general analysis, Phys. Rev. D 69 (2004) 123510 [hep-ph/0402267] [INSPIRE].

[117] Z. Berezhiani, P. Ciarcelluti, D. Comelli and F.L. Villante, Structure formation with mirror dark matter: CMB and LSS, Int. J. Mod. Phys. D 14 (2005) 107 [astro-ph/0312605] [INSPIRE].

[118] P. Ciarcelluti, Cosmology with mirror dark matter. 1. Linear evolution of perturbations, Int. J. Mod. Phys. D 14 (2005) 187 [astro-ph/0409630] [InSPIRE].

[119] P. Ciarcelluti, Cosmology with mirror dark matter. 2. Cosmic microwave background and large scale structure, Int. J. Mod. Phys. D 14 (2005) 223 [astro-ph/0409633] [INSPIRE].

[120] Z. Berezhiani, S. Cassisi, P. Ciarcelluti and A. Pietrinferni, Evolutionary and structural properties of mirror star MACHOs, Astropart. Phys. 24 (2006) 495 [astro-ph/0507153] [INSPIRE].

[121] Z.G. Berezhiani and M.Y. Khlopov, Physics of cosmological dark matter in the theory of broken family symmetry (in Russian), Sov. J. Nucl. Phys. 52 (1990) 60 [inSPIRE].

[122] Z.G. Berezhiani and M.Y. Khlopov, Cosmology of Spontaneously Broken Gauge Family Symmetry, Z. Phys. C 49 (1991) 73 [inSPIRE].

[123] Z.G. Berezhiani and M.Y. Khlopov, The Theory of broken gauge symmetry of families (in Russian), Sov. J. Nucl. Phys. 51 (1990) 739 [inSPIRE].

[124] R.K. Leane, Indirect Detection of Dark Matter in the Galaxy, in 3rd World Summit on Exploring the Dark Side of the Universe, Point à Pitre, Guadeloupe Islands (2020)

[arXiv: 2006.00513] [INSPIRE].

[125] M. Cirelli et al., PPPC 4 DM ID: A Poor Particle Physicist Cookbook for Dark Matter Indirect Detection, JCAP 03 (2011) 051 [Erratum ibid. 10 (2012) E01] [arXiv:1012.4515] [INSPIRE].

[126] HESS collaboration, Search for $\gamma$-Ray Line Signals from Dark Matter Annihilations in the Inner Galactic Halo from 10 Years of Observations with H.E.S.S., Phys. Rev. Lett. 120 (2018) 201101 [arXiv: 1805.05741] [INSPIRE].

[127] H.E.S.S. collaboration, Dark matter search in the inner galactic center halo with H.E.S.S, in 51st Rencontres de Moriond on Cosmology, La Thuile Italy (2016), pg. 149 [arXiv: 1608.08453] [INSPIRE].

[128] H. Aghai-Khozani et al., First measurement of the antiproton-nucleus annihilation cross section at $125 \mathrm{keV}$, Hyperfine Interact. 234 (2015) 85.

[129] A. Bianconi et al., Measurement of the antiproton-nucleus annihilation cross section at 5.3 MeV, Phys. Lett. B $\mathbf{7 0 4}$ (2011) 461 [InSPIRE].

[130] C.J. Batty, E. Friedman and A. Gal, Unified optical model approach to low-energy anti-proton annihilation on nuclei and to anti-protonic atoms, Nucl. Phys. A 689 (2001) 721 [nucl-th/0010006] [INSPIRE].

[131] D. Aristizabal Sierra, F. Staub and A. Vicente, Shedding light on the $b \rightarrow s$ anomalies with a dark sector, Phys. Rev. D 92 (2015) 015001 [arXiv:1503. 06077] [INSPIRE].

[132] G. Bélanger, C. Delaunay and S. Westhoff, A Dark Matter Relic From Muon Anomalies, Phys. Rev. D 92 (2015) 055021 [arXiv:1507.06660] [INSPIRE]. 
[133] B. Allanach, F.S. Queiroz, A. Strumia and S. Sun, $Z^{\prime}$ models for the LHCb and $g-2$ muon anomalies, Phys. Rev. D 93 (2016) 055045 [Erratum ibid. 95 (2017) 119902] [arXiv: 1511.07447] [INSPIRE].

[134] M. Bauer and M. Neubert, Flavor anomalies, the $750 \mathrm{GeV}$ diphoton excess, and a dark matter candidate, Phys. Rev. D 93 (2016) 115030 [arXiv:1512.06828] [InSPIRE].

[135] A. Celis, W.-Z. Feng and M. Vollmann, Dirac dark matter and $b \rightarrow s \ell^{+} \ell^{-}$with U(1) gauge symmetry, Phys. Rev. D 95 (2017) 035018 [arXiv:1608.03894] [inSPIRE].

[136] W. Altmannshofer, S. Gori, S. Profumo and F.S. Queiroz, Explaining dark matter and B decay anomalies with an $L_{\mu}-L_{\tau}$ model, JHEP 12 (2016) 106 [arXiv:1609.04026] [INSPIRE].

[137] P. Ko, T. Nomura and H. Okada, A flavor dependent gauge symmetry, Predictive radiative seesaw and LHCb anomalies, Phys. Lett. B 772 (2017) 547 [arXiv:1701.05788] [InSPIRE].

[138] P. Ko, T. Nomura and H. Okada, Explaining $B \rightarrow K^{(*)} \ell^{+} \ell^{-}$anomaly by radiatively induced coupling in $\mathrm{U}(1)_{\mu-\tau}$ gauge symmetry, Phys. Rev. D 95 (2017) 111701 [arXiv:1702.02699] [INSPIRE].

[139] J.M. Cline, J.M. Cornell, D. London and R. Watanabe, Hidden sector explanation of B-decay and cosmic ray anomalies, Phys. Rev. D 95 (2017) 095015 [arXiv:1702.00395] [INSPIRE].

[140] F. Sala and D.M. Straub, A New Light Particle in B Decays?, Phys. Lett. B 774 (2017) 205 [arXiv: 1704.06188] [INSPIRE].

[141] J. Ellis, M. Fairbairn and P. Tunney, Anomaly-Free Models for Flavour Anomalies, Eur. Phys. J. C 78 (2018) 238 [arXiv:1705.03447] [InSPIRE].

[142] J. Kawamura, S. Okawa and Y. Omura, Interplay between the $b \rightarrow$ sll anomalies and dark matter physics, Phys. Rev. D 96 (2017) 075041 [arXiv: 1706. 04344] [INSPIRE].

[143] S. Baek, Dark matter contribution to $b \rightarrow s \mu^{+} \mu^{-}$anomaly in local $\mathrm{U}(1)_{L_{\mu}-L_{\tau}}$ model, Phys. Lett. B 781 (2018) 376 [arXiv: 1707.04573] [INSPIRE].

[144] J.M. Cline, $B$ decay anomalies and dark matter from vectorlike confinement, Phys. Rev. D 97 (2018) 015013 [arXiv:1710.02140] [INSPIRE].

[145] J.M. Cline and J.M. Cornell, $R\left(K^{(*)}\right)$ from dark matter exchange, Phys. Lett. B 782 (2018) 232 [arXiv: 1711.10770] [INSPIRE].

[146] L. Dhargyal, A simple model to explain observed muon sector anomalies and small neutrino masses, J. Phys. G 46 (2019) 125002 [arXiv:1711.09772] [INSPIRE].

[147] C.-W. Chiang and H. Okada, A simple model for explaining muon-related anomalies and dark matter, Int. J. Mod. Phys. A 34 (2019) 1950106 [arXiv:1711.07365] [INSPIRE].

[148] A. Vicente, Anomalies in $b \rightarrow s$ transitions and dark matter, Adv. High Energy Phys. 2018 (2018) 3905848 [arXiv: 1803.04703] [INSPIRE].

[149] A. Falkowski, S.F. King, E. Perdomo and M. Pierre, Flavourful $Z^{\prime}$ portal for vector-like neutrino Dark Matter and $R_{K^{(*)}}$, JHEP 08 (2018) 061 [arXiv:1803.04430] [INSPIRE].

[150] G. Arcadi, T. Hugle and F.S. Queiroz, The Dark $L_{\mu}-L_{\tau}$ Rises via Kinetic Mixing, Phys. Lett. B $\mathbf{7 8 4}$ (2018) 151 [arXiv: 1803.05723] [INSPIRE].

[151] S. Baek and C. Yu, Dark matter for $b \rightarrow s \mu^{+} \mu^{-}$anomaly in a gauged $\mathrm{U}(1)_{X}$ model, JHEP 11 (2018) 054 [arXiv:1806.05967] [INSPIRE]. 
[152] A. Azatov, D. Barducci, D. Ghosh, D. Marzocca and L. Ubaldi, Combined explanations of B-physics anomalies: the sterile neutrino solution, JHEP 10 (2018) 092 [arXiv: 1807.10745] [INSPIRE].

[153] B. Barman, D. Borah, L. Mukherjee and S. Nandi, Correlating the anomalous results in $b \rightarrow s$ decays with inert Higgs doublet dark matter and muon $(g-2)$, Phys. Rev. D 100 (2019) 115010 [arXiv:1808.06639] [INSPIRE].

[154] D.G. Cerdeño, A. Cheek, P. Martín-Ramiro and J.M. Moreno, B anomalies and dark matter: a complex connection, Eur. Phys. J. C 79 (2019) 517 [arXiv:1902.01789] [INSPIRE].

[155] S. Trifinopoulos, $B$-physics anomalies: The bridge between $R$-parity violating supersymmetry and flavored dark matter, Phys. Rev. D 100 (2019) 115022 [arXiv: 1904.12940] [INSPIRE].

[156] L. Da Rold and F. Lamagna, A vector leptoquark for the B-physics anomalies from a composite GUT, JHEP 12 (2019) 112 [arXiv:1906.11666] [INSPIRE].

[157] J. Fuentes-Martín, M. Reig and A. Vicente, Strong CP problem with low-energy emergent QCD: The 4321 case, Phys. Rev. D 100 (2019) 115028 [arXiv:1907.02550] [InSPIRE].

[158] Z.-L. Han, R. Ding, S.-J. Lin and B. Zhu, Gauged U(1) $)_{L_{\mu}-L_{\tau}}$ scotogenic model in light of $R_{K^{(*)}}$ anomaly and AMS-02 positron excess, Eur. Phys. J. C 79 (2019) 1007 [arXiv: 1908.07192] [INSPIRE].

[159] D. Huang, A.P. Morais and R. Santos, Anomalies in B-meson decays and the muon $g-2$ from dark loops, Phys. Rev. D 102 (2020) 075009 [arXiv:2007.05082] [INSPIRE].

[160] S. Samuel, Bosonic technicolor, Nucl. Phys. B 347 (1990) 625 [InSPIRE]. 